\title{
STELLAR POPULATIONS FOUND IN THE CENTRAL KILOPARSEC OF FOUR LUMINOUS COMPACT BLUE GALAXIES AT INTERMEDIATE $z^{1}$
}

\author{
C. Hoyos, ${ }^{2}$ R. Guzmán, ${ }^{3}$ A. I. Díaz, ${ }^{2,4}$ D. C. Koo, ${ }^{5}$ and M. A. Bershady ${ }^{6}$ \\ Received 2007 February 20; accepted 2007 August 13
}

\begin{abstract}
We investigate the star formation history of the central regions of four luminous compact blue galaxies (LCBGs) at intermediate redshift using evolutionary population synthesis techniques. LCBGs are blue $(B-V \leq 0.6)$, compact $\left(\mu_{B} \leq 21.0 \mathrm{mag} \operatorname{arcsec}^{-2}\right)$ galaxies with absolute magnitudes $M_{B}$ brighter than -17.5 . The LCBGs analyzed here are located at $0.436 \leq z \leq 0.525$. They are among the most luminous $\left(M_{B}<-20.5\right)$, blue $(B-V \leq 0.4)$, and high surface brightness $\left(\mu_{B} \leq 19.0 \mathrm{mag} \operatorname{arcsec}^{-2}\right)$ of this population. The observational data used were obtained with the Hubble Space Telescope (HST) STIS, the WFPC2, and the first NICMOS camera. We have disentangled the stellar generations found in the central regions of the observed targets using a very simple model. This is one of the first times this has been done for compact galaxies at this redshift using HST data, and it provides a comparison benchmark for future work on this kind of galaxy using instruments with adaptive optics in $10 \mathrm{~m}$ class telescopes. We find evidence for multiple stellar populations. One of them is identified as the ionizing population, and the other corresponds to the underlying stellar generation. The estimated masses of the inferred stellar populations are compatible with the dynamical masses, which are typically $(2-10) \times 10^{9} M_{\odot}$. Our models also indicate that the first episodes of star formation these LCBGs underwent happened between 5 and $7 \mathrm{Gyr}$ ago. We compare the stellar populations found in LCBGs with the stellar populations present in bright, local $\mathrm{H}$ in galaxies, nearby spheroidal systems, and blue compact dwarf galaxies. It turns out that the underlying stellar populations of LCBGs are similar to yet bluer than those of local $\mathrm{H}$ in galaxies. It is also the case that the passive color evolution of the LCBGs could convert them into local spheroidal galaxies if no further episode of star formation takes place. Our results help to impose constraints on evolutionary scenarios for the population of LCBGs found commonly at intermediate redshifts.
\end{abstract}

Key words: galaxies: abundances — galaxies: evolution — galaxies: high-redshift — galaxies: stellar content

Online material: color figures

\section{INTRODUCTION}

Luminous compact blue galaxies (LCBGs) are luminous $\left(M_{B}\right.$ brighter than -17.5$)$, blue $(B-V \leq 0.6)$, and compact $\left(\mu_{B} \leq\right.$ $21.0 \mathrm{mag} \operatorname{arcsec}^{-2}$ ) systems experiencing an intense episode of star formation, with typical star formation rates (SFRs) ranging from 1 to $5 M_{\odot} \mathrm{yr}^{-1}$. Although these SFRs are common in other starburst systems, the star formation episode takes place in a very small volume, allowing these compact objects to be seen from cosmological distances. LCBGs are believed to contribute up to $\sim 50 \%$ of the SFR density in the universe at $z \approx 1$ (Guzmán et al. 1997). Their rapid evolution from those times until the present day makes them responsible for the observed evolution of the luminosity function (Lilly et al. 1995), as well as the evolution of the SFR in the universe.

Despite their importance, their relation to local galaxies is poorly known. The work presented in Guzmán et al. (1997), Phillips et al. (1997), and Hoyos et al. (2004) gives a glimpse of the similarities

\footnotetext{
${ }^{1}$ Based on observations obtained with the NASA/ESA Hubble Space Telescope through the Space Telescope Science Institute, which is operated by the Association of Universities for Research in Astronomy (AURA), Inc., under NASA contract NAS5-26555.

2 Departamento de Física Teórica (C-XI), Universidad Autónoma de Madrid, Carretera de Colmenar Viejo km 15.600, 28049 Madrid, Spain; charly.hoyos@ uam.es, angeles.diaz@uam.es.

${ }^{3}$ Bryant Space Science Center, University of Florida, Gainesville, FL 326112055, USA; guzman@astro.ufl.edu.

${ }^{4}$ On sabbatical at the Institute of Astronomy, Cambridge, UK.

5 Department of Astronomy, University of California, Santa Cruz, CA 95064, USA; koo@ucolick.org.

6 Department of Astronomy, University of Wisconsin, Madison, WI 53706, USA; bershady@astro.wisc.edu.
}

between LCBGs and local $\mathrm{H}$ II and starburst nuclei populations. $\mathrm{H}$ II galaxies are a subset of blue compact galaxies (BCGs) in which the spectrum is completely dominated by a component, present almost everywhere within the galaxy, resembling the emission of a H II region (Sargent \& Searle 1970). BCGs were first identified by Zwicky (1965) as faint starlike field galaxies on Palomar Sky Survey plates. BCGs often present an emission-line spectrum and a UV excess. Starburst nuclei were introduced in Balzano (1983); they show high extinction values, with very low [N II] $\lambda 6584 / \mathrm{H} \alpha$ ratios and faint [O III] $\lambda 5007$ emission. Their $\mathrm{H} \alpha$ luminosities are always greater than $10^{8} L_{\odot}$. One of the main ingredients in any theory attempting to explain the final destiny of LCBGs is bound to be an accurate knowledge of the stellar generations already existing in these distant sources. The similarities found between LCBGs and many local $\mathrm{H}$ II galaxies make it possible that the star formation histories of both types of objects are very similar. Even though $\mathrm{H}$ II galaxies were originally thought to be truly young objects, experiencing their very first star formation episodes (Searle \& Sargent 1972; Sargent \& Searle 1970), this hypothesis is no longer tenable after very deep CCD imaging of such objects (Telles \& Terlevich 1997; Telles et al. 1997) or other very similar sources like the blue compact dwarf galaxies (BCDGs). BCDGs are similar to BCGs or $\mathrm{H}$ II galaxies but are an order of magnitude less luminous. The underlying stellar populations of BCDGs are also older than those of BCGs (Papaderos et al. 1996a, 1996b; Loose \& Thuan 1986), which show clear evidence of an older extended population of stars. The properties of this more extended component have been further studied photometrically (see, e.g., Caon et al. 2005; Telles et al. 1997). The current view on local $\mathrm{H}$ II galaxies is that they are a mixture of three populations. The first of them is the youngest 
TABLE 1

Log of HST STIS, WFPC2, AND NIC1 OBSERVATIONS

\begin{tabular}{|c|c|c|c|c|}
\hline Parameter & SA57-7042 & SA57-5482 & SA57-10601 & H1-13088 \\
\hline R.A. ${ }^{\mathrm{a}} \ldots$ & 130726.3 & 130908.8 & 130847.8 & 172019.67 \\
\hline 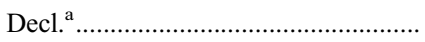 & 291825.7 & 291557.7 & 292341.1 & 500104.7 \\
\hline 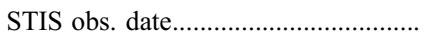 & 2002 May 5 & 2002 Apr 9 & 2000 Jul 5 & 2000 Nov 6 \\
\hline 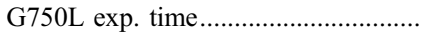 & 9898 & $\ldots$ & 4696 & 4932 \\
\hline 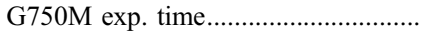 & 2760 & 18106 & 5571 & 5947 \\
\hline G430L exp. time & $\ldots$ & 2790 & $\ldots$ & $\ldots$ \\
\hline G430M exp. time................................ & $\ldots$ & 7709 & $\ldots$ & $\ldots$ \\
\hline WFPC2 F606W exp. time ................. & 700 & 700 & 700 & 700 \\
\hline WFPC2 F814W exp. time ................. & 900 & 900 & 900 & 1000 \\
\hline NICMOS F160W exp. time............... & 2560 & 1280 & 5632 & 1792 \\
\hline Object ID ...................................... & 1 & 2 & 3 & 4 \\
\hline$z$ & 0.525 & 0.453 & 0.438 & 0.436 \\
\hline 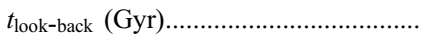 & 5.2 & 4.7 & 4.6 & 4.5 \\
\hline$M_{B}$ & -20.6 & -20.8 & -20.4 & -21.1 \\
\hline 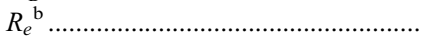 & 1.4 & 1.5 & 1.9 & 2.4 \\
\hline 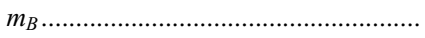 & 22.3 & 21.6 & 21.6 & 21.0 \\
\hline 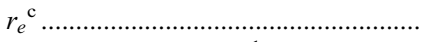 & 0.25 & 0.29 & 0.36 & 0.47 \\
\hline Velocity disp. $\left(\mathrm{km} \mathrm{s}^{-1}\right) \ldots \ldots \ldots \ldots \ldots \ldots \ldots \ldots \ldots \ldots \ldots$ & $120 \pm 10$ & $60 \pm 6$ & $42 \pm 4$ & $47 \pm 4$ \\
\hline $\log M / M_{\odot}{ }^{\mathrm{d}}$ & 10.7 & 10.1 & 9.7 & 9.8 \\
\hline
\end{tabular}

NoTE.-All quantities given were calculated assuming $q_{0}=-0.55$ and $H_{0}=70 \mathrm{~km} \mathrm{~s}^{-1} \mathrm{Mpc}^{-1}$.

a Coordinates are given for equinox J2000.0. Units of right ascension are hours, minutes, and seconds, and units of declination are degrees, arcminutes, and arcseconds.

${ }^{\mathrm{b}}$ Half-light radii in kiloparsecs.

${ }^{c}$ Half-light radii in arcseconds.

${ }^{\mathrm{d}}$ Dynamical masses within $R_{e}$ calculated as in Hoyos et al. (2004). Uncertainties are $20 \%$.

population, responsible for the observed emission lines. The second population is an intermediate-age generation, older than $50 \mathrm{Myr}$ but younger than $1 \mathrm{Gyr}$. This population is unable to ionize the $\mathrm{H}$ I gas, and its luminosity is increasingly dominated by the contribution of giant stars and some asymptotic giant branch stars. The third population is the oldest one, older than 1 Gyr. This population generally dominates the stellar mass by a large factor, and it also occupies a larger volume.

The main target of this study is to characterize the stellar populations present in intermediate-redshift LCBGs by combining the spectral and color information available from these data with evolutionary population synthesis techniques. The modeled stellar populations are then compared to the stellar populations found in $\mathrm{H}$ II galaxies and the smaller yet similar BCDGs. It is also interesting to study the future evolution of the predicted stellar populations, to see whether they can become dwarf elliptical systems (dE or Sph), as suggested by Koo et al. (1995) and Guzmán et al. (1998), or, alternatively, they can be the bulges of spiral galaxies still in the formation process (Hammer et al. 2001; Kobulnicky \& Zaritsky 1999; Guzmán et al. 1998; Phillips et al. 1997).

The observations and analysis techniques, together with the different results they yield, are described in $\S 2$. Section 3 presents the data analysis. The models that have been constructed to explain the observations are discussed in $\S 4$. The LCBGs presented in this work are compared to relevant samples of local galaxies in $\S 5$. The summary is given in $\S 6$.

The cosmology assumed here is a flat universe with $\Omega_{\Lambda}=0.7$ and $\Omega_{m}=0.3$. The resulting cosmological parameters are $H_{0}=$ $70 \mathrm{~km} \mathrm{~s}^{-1} \mathrm{Mpc}^{-1}$ and $q_{0}=-0.55$. Given these values, $1^{\prime \prime}$ corresponds to $1.8 \mathrm{kpc}$ at $z=0.1$ and $5.8 \mathrm{kpc}$ at $z=0.45$.

\section{OBSERVATIONS AND DATA REDUCTION}

In this paper we present long-slit spectra, deep optical $V$ (F606W) and $I(\mathrm{~F} 814 \mathrm{~W})$ images, and infrared F160W images of four LCBGs with redshifts between 0.436 and 0.525 . The data were obtained using the Space Telescope Imaging Spectrograph (STIS), the WFPC2, and the first NICMOS camera. Target names, redshifts, look-back times, $B$ absolute magnitudes, exposure times, halflight radii, velocity dispersions, and virial masses within $R_{e}$, as well as an identifying numeral for the observed objects, are given in Table 1.

The STIS long-slit spectra are imaged on a SITe $1024 \times 1024$ CCD, with $0.05^{\prime \prime}$ square pixels operating from $\sim 2000$ to $11000 \AA$. Several instrumental configurations were used. The instrumental setup used to observe objects 1,3 , and 4 used the low-resolution grating G750L. This instrumental setup provides a dispersion of $4.92 \AA$ pixel $^{-1}$, or about $190 \mathrm{~km} \mathrm{~s}^{-1}$ pixel $^{-1}$, which in combination with a slit $0.5^{\prime \prime}$ wide gives a spectral resolution of about $29 \AA$ (FWHM). The spectral coverage provided by this setup is about $5000 \AA$. The central wavelength is $7750 \AA$. The higher resolution grating G750M was also used for objects 3 and 4 . It provides a dispersion of $0.53 \AA$ pixel $^{-1}$, or about $35 \mathrm{~km} \mathrm{~s}^{-1}$ $\mathrm{pixel}^{-1}$. The slit width used was $0.2^{\prime \prime}$, thus giving a spectral resolution of about $2.1 \AA$ (FWHM). The spectral coverage provided by this setup is about $500 \AA$. These spectra are used to study the gas-phase velocity field, and the results from this work are presented in Bershady et al. (2005). Object 2 was observed using the lower resolution grating $\mathrm{G} 430 \mathrm{M}$ and the high-resolution grating G750M. The G430M grating has a dispersion of $2.7 \AA$ pixel $^{-1}$, and the slit width used is $0.2^{\prime \prime}$. The central wavelength is $4300 \AA$, and the spectral coverage is $2800 \AA$. The data were reduced using the STSDAS $^{7}$ package within IRAF. ${ }^{8}$ For a complete account of the reduction procedures and uncertainties and detailed descriptions of the spectra, see Hoyos et al. (2004). It is worth mentioning that

\footnotetext{
${ }^{7}$ STSDAS is the Space Telescope Science Data Analysis System from the Space Telescope Science Institute, operated for NASA by AURA, Inc.

${ }^{8}$ IRAF is the Image Reduction and Analysis Facility, distributed by the National Optical Astronomy Observatory, which is operated by AURA, Inc., under cooperative agreement with the National Science Foundation.
} 

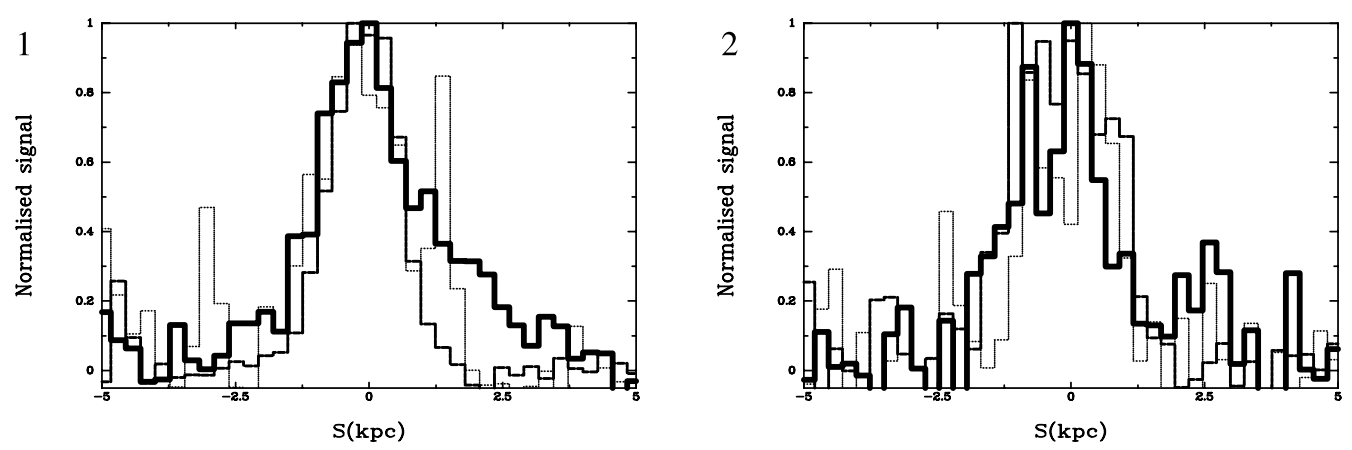

FIG. 1.-Continuum and emission-line spatial profiles. Only objects 1 and 2 are presented, since the spatial profiles for objects 3 and 4 are given in Hoyos et al. (2004). The $S$-axis is placed with respect to the slits depicted in Fig. 2. Increasing $S$ corresponds to upper positions in that figure. The continuum is shown by the thick solid line, $[\mathrm{O}$ III $] 25007$ is shown by the thin solid line, and the dotted line represents $\mathrm{H} \beta$.

STIS spectroscopic observations using the G750L and G750M gratings are affected by fringing in the red end of the spectrum. This makes it necessary to use specially designed flats (called "contemporaneous flats") in order to circumvent this issue. The use of this technique, which is implemented as a task within STSDAS, raises the signal-to-noise ratio $(\mathrm{S} / \mathrm{N})$ by around $15 \%$. This is crucial for these observations. The proposal IDs for these observations are GO 8678 and GO 9126.

The images used were taken with the WFPC2, with the LCBGs centered in the Planetary Camera, a $800 \times 800$ CCD, with $0.046^{\prime \prime}$ square pixels. Two broadband filters were used, the F814W filter and the F606W filter. For each object and filter, two $\sim 400$ s exposures were obtained, to allow for cosmic-ray rejection. The details of the reduction and analysis procedures can be found in Guzmán et al. (1998). The proposal ID for these data is GO 5994.

The infrared frames were obtained with the NIC1 camera of NICMOS. ${ }^{9}$ This camera uses a low-noise, high quantum efficiency, $256 \times 256$ pixel $\mathrm{HgCdTe}$ array, with $0.0431^{\prime \prime}$ square pixels. The filter used was the F160W, which has a bandpass of around $4000 \AA$. For each object, several different exposures were collected and combined to produce a final mosaic. The proposal ID is GO 7875 .

The STIS data can only probe the central regions of these galaxies because their outermost regions are too faint to be studied spectroscopically with the Hubble Space Telescope (HST). Specifically, the STIS data can be used to study the line-emitting region, where the starburst is taking place and most of the gas is ionized. The line-emitting region of the observed galaxies has a radius between 1.4 and $2.0 \mathrm{kpc}$. This is a little bit less than one effective radius. On the other hand, the WFPC 2 images and NICMOS frames are deeper, and the size of the observed galaxies in the images is much larger than the size of the line-emitting region in the STIS spectra. The analysis presented in this paper focuses on this particular central region where the STIS spectra spatially overlap with the WFPC2 images and NICMOS frames. This region has a radius of $1.7 \mathrm{kpc}$, on average.

\section{RESULTS AND ANALYSIS}

\subsection{Emission-Line and Continuum Distribution}

It is interesting to investigate whether the continuum distribution and emission-line distribution differ in the STIS spectra. This will help determine where the starburst has taken place. This information can be helpful in understanding the starburst triggering mechanism and in constructing models for these objects. Here we follow the analysis presented in Hoyos et al. (2004), although

\footnotetext{
${ }^{9}$ Before the cryo-cooler was installed.
}

it is given in a much simpler way. Furthermore, only objects 1 and 2 are presented here, since the other two sources were dealt with in Hoyos et al. (2004). The procedure is briefly reviewed.

Using the IRAF task fitld, a "continuum frame" was made by fitting three cubic spline pieces to every line in the spectra; then a "line frame" was constructed by subtracting the continuum frame from the original two-dimensional spectroscopic frame. The spatial distribution of the continuum is obtained by columnaveraging the whole continuum frame, and the spatial distributions of the emission lines are obtained by column averaging the line frame over the columns that span 1.25 times the FWHM of the line. The spatial profiles for the two new objects studied are given in Figure 1.

Figure 1 shows the following:

1. The spatial distribution of the ionized gas and underlying stellar population in object 1 is very similar to the distributions observed in objects 3 and 4 (Hoyos et al. 2004). The nebular component light centroid does not coincide with the continuum centroid, although the peaks of the different components clearly line up. The two peaks observed at +1.5 and $-2.7 \mathrm{kpc}$ in the $\mathrm{H} \beta$ spatial profile are not real. This object is very similar to the cometary BCDGs. In this particular case, the spread of the continuum is about twice the size of the line-emitting region. In the optical images, this object shows a conspicuous tail and a very bright knot. It is also seen that the spatial extents of the two emission lines used to trace the ionized gas are very similar.

2. For object 2, the differences in the centroid positions for the continuum and the line-emitting region are very small when compared to the spread of both. In this case, the ionized gas can be said to lie in the center of the optical galaxy. This is to say that the line-emitting region coincides with the continuum light centroid. The true barycenter of these galaxies is not known. It is also interesting to note that, in this particular case, the distributions of each component are very noisy even for the continuum, and that the distributions of $\mathrm{H} \beta$ and [O III] $\lambda 5007$ are very similar to each other.

As in Hoyos et al. (2004), the $\mathrm{H} \beta$ and [O III] $\lambda 5007$ lines were chosen to probe the ionized gas phase because [O $\mathrm{III}] \lambda 5007$ and $\mathrm{H} \beta$ are very close to each other in wavelength space, and therefore they are affected by a similar extinction. For this reason, differences in their spreads can then reveal real differences in their distributions, not just a reddening effect. As pointed out in Hoyos et al. (2004), the observed differences in the spread of $\mathrm{H} \alpha$ and $\mathrm{H} \beta$ or $[\mathrm{O}$ III] $\lambda 5007$ and [O II] $\lambda 3727$ can be accounted for by moderate extinction, within the measurement errors, making it impossible to detect extinction or excitation gradients unambiguously. Finally, the inclusion of these two new objects in the 
TABLE 2

Absorption Coefficients, Dereddened Line Ratios, Rest-Frame Equivalent Widths, Oxygen Content, and Stellar Colors

\begin{tabular}{|c|c|c|c|c|c|c|c|c|}
\hline \multirow[b]{2}{*}{ PARAMETER } & \multicolumn{2}{|c|}{ ОвЈест 1} & \multicolumn{2}{|c|}{ ОвЈест 2} & \multicolumn{2}{|c|}{ Овјест 3} & \multicolumn{2}{|c|}{ Овјест 4} \\
\hline & Inner & Outer & Inner & Outer & Inner & Outer & Inner & Outer \\
\hline Size $(\mathrm{kpc})$, & 0.83 & 1.66 & 1.04 & 2.01 & 1.28 & 2.03 & 1.27 & 3.04 \\
\hline$c(\mathrm{H} \beta)$ & $0.0 \pm 0.1$ & $0.0 \pm 0.1$ & $0.0 \pm 0.1$ & $0.0 \pm 0.2$ & $0 \pm 0.1$ & $0.10 \pm 0.2$ & $0.10 \pm 0.05$ & $0.10 \pm 0.05$ \\
\hline 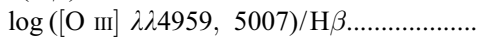 & $0.71 \pm 0.04$ & $0.60 \pm 0.03$ & $0.60 \pm 0.03$ & $0.39 \pm 0.04$ & $0.67 \pm 0.03$ & $0.44 \pm 0.06$ & $0.7 \pm 0.1$ & $0.54 \pm 0.08$ \\
\hline $\log \left(\left[\mathrm{O}_{\mathrm{III}}\right] /\left[\mathrm{O}_{\mathrm{II}}\right]\right)^{\mathrm{a}} \ldots \ldots \ldots \ldots \ldots \ldots \ldots$ & $0.48 \pm 0.04$ & $0.38 \pm 0.02$ & $-0.31 \pm 0.04$ & $-0.38 \pm 0.05$ & $0.43 \pm 0.06$ & $0.33 \pm 0.09$ & $0.28 \pm 0.09$ & $0.26 \pm 0.07$ \\
\hline $\log R_{23} \mathrm{~b} \ldots \ldots$ & $0.83 \pm 0.04$ & $0.75 \pm 0.02$ & $1.08 \pm 0.02$ & $0.92 \pm 0.06$ & $0.80 \pm 0.03$ & $0.61 \pm 0.05$ & $0.89 \pm 0.08$ & $0.73 \pm 0.06$ \\
\hline $\log W_{\left[\mathrm{O} \text { II] } \lambda 3727^{\mathrm{c}}\right.} \ldots \ldots$ & 1.88 & 1.94 & 2.18 & 2.11 & 1.65 & 1.43 & 1.93 & 1.79 \\
\hline $\log W_{\mathrm{H} \beta}{ }^{\mathrm{c}} \ldots \ldots \ldots$ & 1.79 & 1.87 & 0.60 & 0.47 & 1.59 & 1.51 & 1.67 & 1.57 \\
\hline $\log W_{[\mathrm{O} \text { II] }]} \lambda \lambda 4959+5007^{\mathrm{c}}$ & 2.51 & 2.53 & 1.10 & 0.77 & 2.28 & 2.00 & 2.40 & 2.13 \\
\hline $\log W_{\mathrm{H} \alpha} \mathrm{c}$ & 2.00 & 1.82 & $\ldots$ & $\ldots$ & 2.30 & 2.16 & $\ldots$ & $\ldots$ \\
\hline 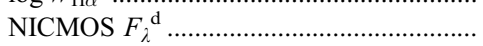 & $2.8 \pm 0.3$ & $\ldots$ & $5.9 \pm 0.6$ & $\ldots$ & $3.9 \pm 0.4$ & $\ldots$ & $10 \pm 1$ & $\ldots$ \\
\hline 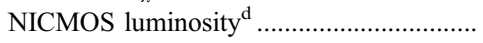 & 2.7 & $\ldots$ & 3.9 & $\ldots$ & 2.4 & $\ldots$ & 7.0 & $\ldots$ \\
\hline $12+\log (\mathrm{O} / \mathrm{H})_{\mathrm{hi}} \mathrm{e}^{\mathrm{e}}$ & 8.4 & 8.5 & 7.8 & 8.1 & 8.4 & 8.7 & 8.2 & 8.5 \\
\hline $12+\log (\mathrm{O} / \mathrm{H})_{\mathrm{lo}} \mathrm{f}$ & 7.8 & 7.7 & 8.8 & 8.6 & 7.7 & 7.5 & 8.0 & 7.7 \\
\hline$B-V_{\mathrm{obs}}^{\mathrm{g}}$ & 0.41 & 0.49 & 0.28 & 0.27 & 0.08 & 0.25 & 0.26 & 0.35 \\
\hline$B-V_{\text {stellar }}{ }^{\mathrm{h}}$ & 0.13 & 0.20 & 0.34 & 0.33 & -0.08 & 0.16 & 0.12 & 0.28 \\
\hline
\end{tabular}

NoтеS.- In the first row the physical sizes of each aperture in linear kiloparsecs are given. For object 1, the logarithmic extinction coefficient was derived from the $\mathrm{H} \gamma / \mathrm{H} \beta$ ratio. Its value was consistent with the value derived using $\mathrm{H} \alpha$, but the error was smaller. For object 2 , the [O II] $\lambda 3727$ flux is first measured in the integrated G430L spectra. The intensities in each zone (a or b) are then derived by requiring that the zones emit $50 \%$ of the light each. This is true to within $5 \%$ for the other three lines seen in the other instrumental setup. The reported values of $\log W_{[\mathrm{O} \text { II] } / 3727}$ also correspond to the integrated spectra. For object $4, \mathrm{H} \alpha$ is not observed and the listed value for the logarithmic extinction coefficient is an assumed value, typical for local $\mathrm{H}$ II galaxies.

a This is defined as the reddening-corrected ratio [O III] $\lambda \lambda 4959,5007 /[\mathrm{O}$ II $] \lambda 3727$. The other line ratios are also reddening-corrected.

${ }^{\mathrm{b}} R_{23}$ is defined as the reddening-corrected ratio $([\mathrm{O} \mathrm{II}] \lambda 3727+[\mathrm{O} \mathrm{III}] \lambda \lambda 4959,5007) / \mathrm{H} \beta$.

c The rest-frame equivalent widths are in angstroms. The average error in the rest-frame equivalent width is $17 \%$. This corresponds to an error of 0.07 in the logarithm. Most errors fall in the $15 \%-20 \%$ interval. The G750M spectra used for object 2 bear the largest uncertainties, since the slit width used was $0.2^{\prime \prime}$ wide, compared to the $0.5^{\prime \prime}$ slit used for the other three objects. The errors in the equivalent widths for object 2 are therefore $30 \%$, corresponding to 0.13 in the logarithm.

${ }^{\mathrm{d}}$ Units of $F_{\lambda}$ are $10^{-15} \mathrm{ergs} \mathrm{s}^{-1} \mathrm{~cm}^{-2}$. The reported luminosities are rest-frame luminosities in the rest-frame wavelength range that shifts to the $\mathrm{F} 160 \mathrm{~W}$ wavelength passband. The luminosity units are $10^{42} \mathrm{ergs} \mathrm{s}^{-1}$, and the errors are typically $15 \%$. Both of these numbers refer to the total luminosity from the line-emitting region.

${ }^{\mathrm{e}}$ Oxygen abundance derived using the calibration for the upper branch (Pilyugin 2000). The solar value is 8.69 (Allende-Prieto et al. 2001).

${ }^{\mathrm{f}}$ Oxygen abundance derived using the calibration for the lower branch (Pilyugin 2000). The solar value is 8.69 (Allende-Prieto et al. 2001).

g Observed colors. The uncertainty is $0.01 \mathrm{mag}$ in all cases.

${ }^{\mathrm{h}}$ Rest-frame, stellar-population-only, extinction-corrected $B-V$ colors. This is defined in the text. The uncertainty is 0.09 mag.

sample of LCBGs with high-quality spatially resolved spectroscopy allows us to give a first interval of the frequency of offcenter $^{10}$ starbursts in LCBGs. Since the result is that three out of six LCBGs with STIS spectroscopy happen to have their starbursts displaced from their centers, all that can be said is that the fraction of LCBGs with shifted starbursts is $50 \%{ }_{-20 \%}^{+49 \%}$. More observational work is needed to assess the frequency and impact of this issue in the evolution of LCBGs.

\subsection{Line Ratios, Reddening, Equivalent Widths, and Metallicities}

The observed flux of each emission line and the equivalent widths have been measured via Gaussian fits using the STSDAS task ngaussfit, according to the method outlined in Hoyos et al. (2004). This task can fit to a set of $x-y$ values the sum of a straight baseline and one or several Gaussian profiles. This task also gives approximate errors for the fitted parameters, calculated by resampling. The emission lines were fitted rather than summed because of residuals of bad pixels.

Two different positions are considered for each galaxy corresponding to the inner (a) and the outer (b) regions of each object. Despite these names, both the $\mathrm{a}$ and $\mathrm{b}$ regions defined here are within the line-emitting region. These two one-dimensional extractions are defined so as to encompass approximately the same line luminosity. It is in these two regions that the following stellar population analysis is carried out. These apertures are near one effective radius for the line-emitting region, as detected through

\footnotetext{
${ }^{10}$ Here the word "center" means the continuum light centroid.
}

the spectroscopic aperture. The linear sizes of both extractions are given in the second row of Table 2, in kiloparsecs. For position a, both the FWHM and central wavelength are left as free parameters. However, for position b, the FWHM is fixed to the FWHM derived for position a to decrease the number of free parameters and hence increase the goodness of the fit in poor $\mathrm{S} / \mathrm{N}$ conditions. The $[\mathrm{O}$ II] $\lambda 3727, \mathrm{H} \beta$, and both [O III] lines can be observed in all cases. However, although $\mathrm{H} \alpha$ is detected for object 4 , it is affected by many bad pixels that render the line unusable. In the case of object $1, \mathrm{H} \gamma$ is detected, too.

The amount of extinction is here parameterized using the logarithmic extinction coefficient $c(\mathrm{H} \beta)$. It was derived using standard nebular analysis techniques (Osterbrock 1989) using the $\mathrm{H} \alpha / \mathrm{H} \beta$ Balmer line ratios. For object 1, the $\mathrm{H} \gamma$ line was used to derive the extinction coefficient, which agreed well with that derived from $\mathrm{H} \alpha$ and $\mathrm{H} \beta$ but had a smaller uncertainty. Since the observed galaxies are at high Galactic latitude, suffering Galactic extinctions of $A_{B}<0.11 \mathrm{mag}$ which correspond to $c(\mathrm{H} \beta)<0.05$ (Burstein \& Heiles 1982; Sandage \& Tamman 1974), it was assumed that all extinction occurs within the target objects. This approximation is further validated by the fact that the incoming light is redshifted, and therefore the extinction will be much lower. Consequently, the spectra have been dereddened in the rest frame of the targets according to a standard reddening curve (Whitford 1958 ), assuming case B recombination theoretical line ratios. The use of different extinction curves will not significantly change the results, since they do not differ from the one used here in the wavelengths of interest. The value of $c(\mathrm{H} \beta)$ is determined independently for the inner and outer regions of all objects but H1-13088, 
for which $\mathrm{H} \alpha$ is unfortunately not well measured. A global value of $c(\mathrm{H} \beta)=0.1 \pm 0.05$, typical for local $\mathrm{H}$ II galaxies (Hoyos \& Díaz 2006), is adopted. No underlying stellar hydrogen absorption is detected, as the continuum is very low.

Line equivalent widths were measured in the STIS spectra. It is possible to measure the line equivalent widths, despite the fact that these spectra are emission-line-dominated, if the errors are carefully estimated. This is explained in detail in Hoyos et al. (2004), where it is also possible to see four examples of the extracted spectra (see Hoyos et al. 2004, Fig. 2).

The measured extinction coefficients and dereddened line ratios are given in Table 2, together with the measured rest-frame equivalent widths. It is seen that the extinction values are always very small. It can also be seen that the ionization ratio $\log [\mathrm{O}$ III $] /[\mathrm{O}$ II $]$ and both the $\log [\mathrm{O} \mathrm{III}] /[\mathrm{H} \mathrm{II}]$ and $\log R_{23}$ numbers are always smaller in the outer zones of the observed galaxies than in the inner part of the line-emitting region. We define $R_{23}$ as the reddeningcorrected $([\mathrm{O}$ II $] \lambda 3727+[\mathrm{O}$ III $] \lambda \lambda 4959,5007) / \mathrm{H} \beta$ ratio. In some cases, the differences are larger than the derived errors, revealing the existence of a gradient. For some other cases the differences are more dubious. However, even though it is not possible to claim that these gradients have been detected for all of the individual objects studied, the fact that these differences between the $\mathrm{a}$ and $\mathrm{b}$ zones hold for the majority of cases allows us to think that these gradients are real, at least for typical LCBGs.

\subsection{The Oxygen Content and Metallicity Estimates}

One of the main goals of any nebular analysis is to estimate the metallicity of the emitting nebula. There are many reasons to determine the metallicity of the ionized gas in a galaxy, but chief among them is that it yields an up-to-date determination of the chemical composition of the most recent generation of stars born in the galaxy studied.

The emission-line spectrum from a star-forming region depends strongly on the electron temperature and metallicity of the nebula. The ratio of the auroral line [O III] $\lambda 4363$ to the lower excitation lines $[\mathrm{O}$ III $] \lambda \lambda 4959,5007$ gives a direct determination of the electron temperature where $\mathrm{O}^{+}$and $\mathrm{O}^{++}$are the dominant species, but beyond certain metallicities, the increasing cooling leaves no energy to collisionally excite the upper levels. When this is the case, one must resort to determining the oxygen abundance empirically.

In principle, the oxygen content can be determined from the intensities of the optical emission lines using the $R_{23}$ number, defined as the reddening-corrected ratio ([O II $] \lambda 3727+$ [O III $] \lambda \lambda 4959,5007) / \mathrm{H} \beta$. This method was originally developed by Pagel et al. (1979; see also Pagel et al. 1980, Edmunds \& Pagel 1984, McGaugh 1991, and Kewley \& Dopita 2002, among many others). The main problem with this empirical method is that the calibration is double-valued, and some a priori knowledge of the metallicity range is needed in order to solve this degeneracy. Since the $\mathrm{S} / \mathrm{N}$ of the spectra used is not very large, no other lines that could help break this degeneracy are detected. Thus, the lack of this initial knowledge about the oxygen content remains, and the metal content of the galaxies studied cannot be cleanly determined. Instead, the metallicity values that result from the use of the Pilyugin (2000) calibration for both the lower and upper branches are calculated. The metallicity estimates calculated using the Pilyugin (2000) formulae are given in Table 2. Unfortunately, the measured values for $\log R_{23}$ squarely place our objects in the turnaround region of the $12+\log (\mathrm{O} / \mathrm{H})-\log R_{23}$ plot. In this regime, the only thing that can be safely said is that the metallicity is between 7.7 and 8.4. Therefore, the analysis can only safely conclude that these LCBGs have an oxygen content greater than 7.7 but lower than the solar value of 8.69 (AllendePrieto et al. 2001). It is also worth mentioning that, with the available data, it is not possible to unambiguously detect a gradient in the metallicity of these objects, since the derived values of $R_{23}$ imply that the oxygen content of both zones can lie in a very wide range. ${ }^{11}$ It can also be concluded that the $R_{23}$ method is not bestsuited to determining the gas-phase metallicity of LCBGs because the $R_{23}$ diagnostic is likely to fall in this regime.

However, one initial metallicity estimate can be provided by the ratio [ $\left.\mathrm{N}_{\mathrm{II}}\right] \lambda 6584 / \mathrm{H} \alpha$, as suggested by Denicoló et al. (2002). Since $[\mathrm{N}$ II] $\lambda 6584$ cannot be measured in the spectra analyzed here, as it is below the detection limit due to poor $\mathrm{S} / \mathrm{N}$, it is not possible to use the spectra used here directly for this task. The only available information is the Guzmán et al. (1996) W. M. Keck telescope $[\mathrm{N}$ II] $\lambda 6584 / \mathrm{H} \alpha$ measurements for a set of very similar sources from the same parent sample. These measurements consist of very high quality spectra obtained using the High Resolution Echelle Spectrometer (HIRES). The average oxygen content of the Guzmán et al. (1996) sources, derived using the Denicoló et al. (2002) expression for $12+\log (\mathrm{O} / \mathrm{H})$, is $12+$ $\log (\mathrm{O} / \mathrm{H})=8.6 \pm 0.1$. This value is a little bit too high to be completely compatible with the majority of the Pilyugin (2000) metallicity calculations presented above, although it is compatible with some of them. This indicates that the oxygen content is much more likely to lie in the upper half of the interval allowed by the oxygen line ratios. It is interesting to note that the HIRES measurements of [ $\mathrm{N}$ II] $\lambda 6584 / \mathrm{H} \alpha$ are all around 0.18 , and that the $3 \sigma$ detection threshold at $\mathrm{H} \alpha$ is around $15 \%$ of $\mathrm{H} \alpha$ for the STIS spectra, meaning that the STIS spectra were very close to detecting the nitrogen line. This is true for both the low-resolution and high-resolution configurations.

\subsection{Scaling and Registering of the Data and Calculation of Stellar Colors and NIR Luminosities}

In order to describe the stellar populations found in the galaxies studied, it is necessary to match the STIS spectroscopic frames with the WFPC2 images and the NICMOS frames. This is done by forcing each galaxy's centroid in the WFPC2 and NICMOS images to coincide with the center of the continuum distribution of the STIS spectroscopic frames. Once both centroids are calculated, the slit orientation with respect to the image is used to determine what pixels in the WFPC2 frames correspond to the inner and outer zones defined previously for the STIS spectra.

Figure 2 shows the slit placement in the F814W and F606W WFPC2 images. The bidimensional spectra presented for objects 1,3 , and 4 are low-resolution G750L frames, while the spectrum presented for object 2 is a high-resolution $\mathrm{G} 750 \mathrm{M}$ frame. In this figure, the spectra have been scaled and approximately aligned with the images. It is seen that the STIS spectra only probe the most luminous regions of the observed objects, corresponding to the line-emitting region. This area spans less than $1^{\prime \prime}$, and it has been further divided into two regions. This makes it necessary to use the spatial resolution of the HST instruments. Even though these objects have been observed from the ground in the UBRIK bands, it is not possible to merge those data with the observations presented here because the spatial resolution of those observations is not high enough to study the presented objects at the subarcsecond level. The stellar population analysis presented in the following section is therefore best suited for the volume within the line-emitting regions of the studied galaxies. This region typically has a radius of $1.7 \mathrm{kpc}$.

\footnotetext{
${ }^{11}$ In addition, the metallicity gradients of compact systems are likely to be intrinsically small because of the mixing.
} 

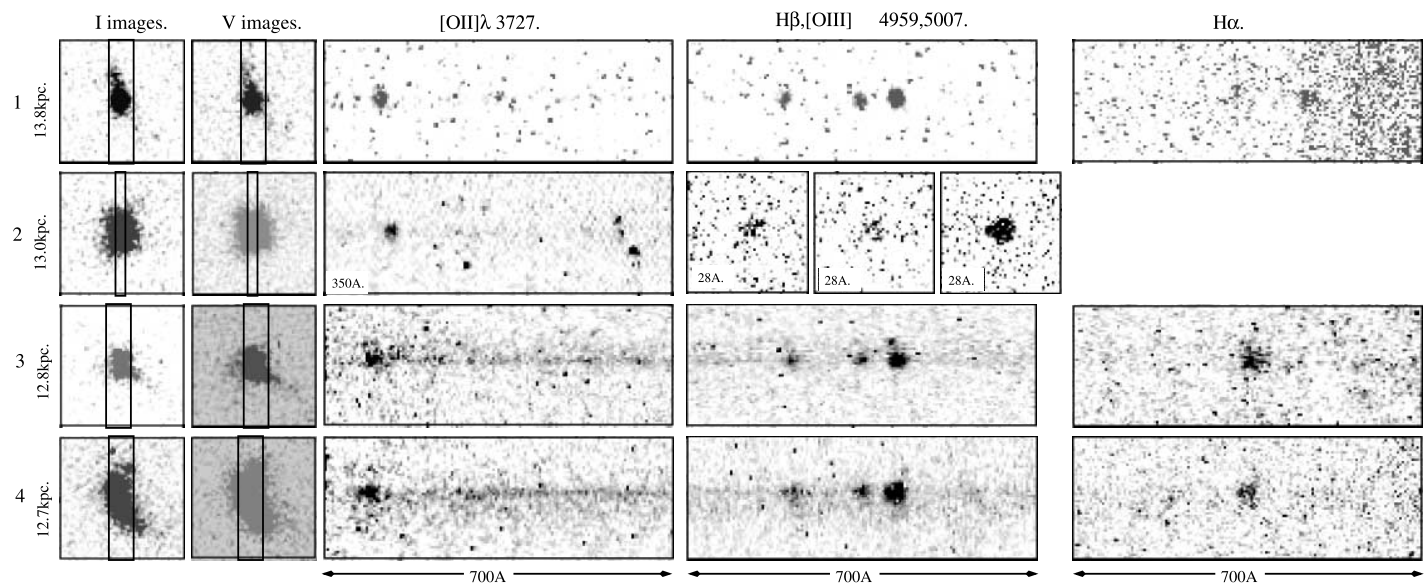

FIG. 2.-Matching between the STIS two-dimensional spectra and the HST WFPC2 images. Each spectral strip spans $2.5^{\prime \prime}$ in the spatial direction. The linear scale is given for each object in the figure. Each spectral strip spans $700 \AA$ except for object 2, in which the wavelength coverage is given in each image. The spectral strips presented for these objects are not registered with respect to the spectral strips shown for the other objects for this reason. The H $\alpha$ strip for object 1 is not perfectly registered with respect to the $\mathrm{H} \alpha$ strips for objects 3 and 4 because the $\mathrm{H} \alpha$ line fell very near the CCD edge. This spectral strip still spans $700 \AA$, however. The slit orientation is shown superimposed on HST F606W and F814W images which are 2.5" on a side. It is interesting to note that the spatial distribution of the continuum is lopsided with respect to the peak. This is most evident in objects 3 and 4.

Figure 3 shows the NICMOS frames. Each panel is $2.5^{\prime \prime}$ on a side. Again, the images have been rotated so that the slit silhouette is vertical, as shown in the figure. It is seen that the STIS spectra focus on the very central regions of the objects.

The next step is to determine the $B-V$ color of the areas in the WFPC2 F814W and F606W images that match the inner (a) and outer (b) zones of the STIS spectra. In order to accurately determine said color, several issues must be taken into account. The first of them is to correct the integrated DN (CCD counts) in each area for the imperfections of the charge transfer mechanism. There are several options to accomplish this task. The version used in the WFPC2 Instrument Handbook, based on the 2002 May 31 version of A. Dolphin's calculations, was used. This correction depends on the objects' location in the CCD. It also depends on the objects' accumulated DN and CCD gain. This correction is
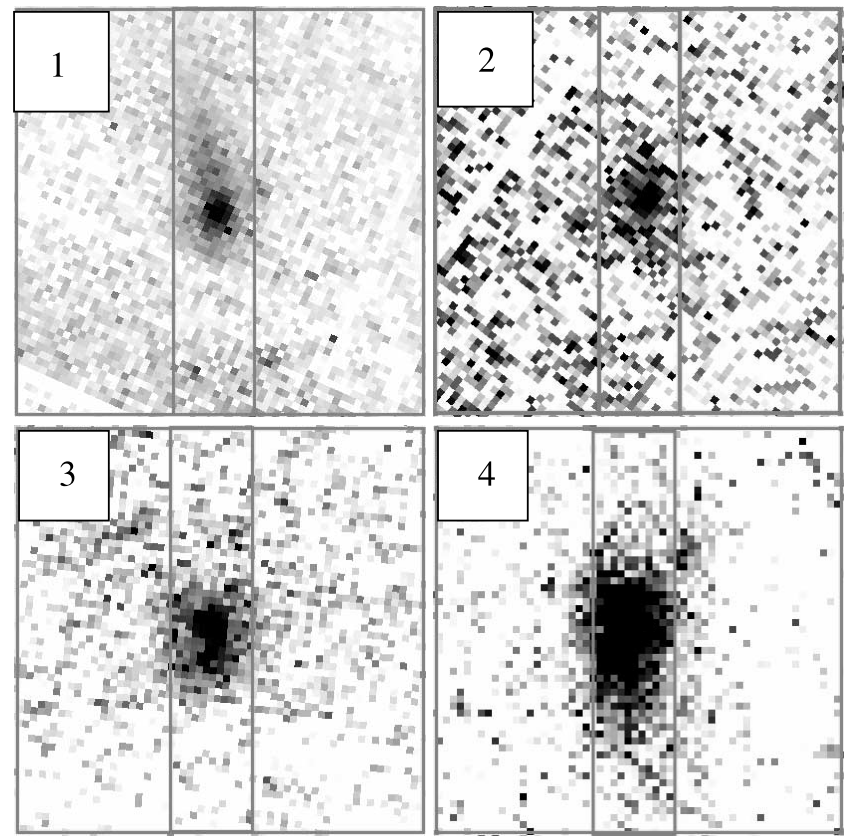

FIG. 3.-NICMOS F160W reduced images. Each postage stamp is $2.5^{\prime \prime}$ on a side. The slit is placed as in Fig. 2. The number in the upper left corner of each panel corresponds to the identifying numeral of Table 1. obviously insensitive to the filter used. Fortunately, for the observations used, any given galaxy is always imaged in the same CCD location, and the accumulated counts are very similar for the two filters used. This correction is therefore very small. It only changes the observed colors by less than $0.1 \mathrm{mag}$, usually much less. The statistical error introduced by this correction was estimated by varying the DN parameter of the software tool previously mentioned around the observed DN number. It turned out that the magnitude error introduced by this correction was negligibly small, $0.001 \mathrm{mag}$ in the worst case. For a complete review of this problem and the different approaches to correct for it, see Dolphin (2000).

The second issue is the derivation of the $k$-correction. This step is needed to convert from the observed $I-V$ colors to the rest-frame $B-V$ colors. The fits given in Fukugita et al. (1995) for the case of an irregular Magellanic spectrum are used. The $k$-corrections are 0.32 for objects 3 and $4,0.39$ for object 2 , and 0.47 for the furthest object. The errors introduced by this correction are mainly systematic, arising from deviations of the real galaxy spectra from the assumed Irr spectrum. These are estimated to be around $0.08 \mathrm{mag}$, as was done in Guzmán et al. (1998).

The third issue is to derive the emission-line-free colors. The method presented in Telles \& Terlevich (1997) is adopted. This method uses the rest-frame equivalent widths, together with the $B$ and $V$ filter transfer functions, to estimate the contribution of the emission lines to the observed galaxy colors. The method is now sketched. Given an observed color $A-B$, it can be written as

$$
B-A=C_{B A}-2.5 \log \frac{L_{B}^{c}+L_{B}^{l}}{L_{A}^{c}+L_{A}^{l}}
$$

where $C_{B A}$ is the photometric constant, $L_{X}^{c}$ is the continuum luminosity in the $X$ band, and $L_{X}^{l}$ is the line luminosity falling within the $X$ band.

If the line luminosities are considered to be small perturbations to the spectrum, the above equation can be cast into the following form:

$$
B-A=(B-A)_{0}+1.086\left(L_{A}^{l} / L_{A}^{c}-L_{B}^{l} / L_{B}^{c}\right),
$$


where $(B-A)_{0}$ is the color that would be observed in the absence of the emission lines.

It is also possible to write the color correction as

$$
A-B=(A-B)_{0}+1.086\left(\frac{L_{A}^{l}}{I_{A} W_{A}}-\frac{L_{B}^{l}}{I_{B} W_{B}}\right),
$$

where $W_{X}$ is the bandpass of the $X$ band and $I_{X}$ is the average specific intensity.

The color correction can therefore be written as

$$
1.086\left[\frac{\sum A_{i} \operatorname{EW}(i)}{W_{A}}-\frac{\sum B_{i} \operatorname{EW}(i)}{W_{B}}\right],
$$

where $\operatorname{EW}(i)$ is the line equivalent width of the $i$ th emission line and $X_{i}$ is the filter transfer function evaluated at that wavelength.

It is important to note that, even if the line equivalent widths used in the above formulae bear large uncertainties, any error in the measured equivalent widths is greatly diminished or canceled out. This happens because the same equivalent width appears in both terms of the color correction with opposite signs, although with possibly different multiplicative factors.

The applied corrections range from essentially zero to $0.25 \mathrm{mag}$. The statistical error introduced by this correction is very small. Given the derived uncertainties in the measured equivalent widths, these errors are less than 0.02 mag.

Finally, the $E(B-V)$ color excess is derived from the $c(\mathrm{H} \beta)$ values for each zone. These color excesses are very small and virtually error-free. The resulting colors are therefore rest-frame, stellar-population-only, extinction-corrected $B-V$ colors. The total photometric errors, obtained by adding in quadrature all the aforementioned uncertainties, are estimated to be 0.09 for all objects. These colors are gathered in Table 2 for the inner and outer parts of the line-emitting region in which the stellar populations are studied.

The NICMOS data were used to calculate the total F160W luminosities of the line-emitting regions. It is necessary to use the NICMOS data in this way because of the larger angular extent of the NICMOS point-spread function, which makes it impossible to calculate the observed fluxes of the $a$ and $b$ zones separately with the same accuracy as for the optical frames. The measured fluxes and luminosities are gathered in Table 2. The reported luminosities all assume $c(\mathrm{H} \beta)=0.2$, and they also assume that $A_{X} / A_{V}=0.4$, where $A_{X}$ is the extinction in the wavelength range that maps into the $\mathrm{F} 160 \mathrm{~W}$ passband. This value is the average value for that ratio between the $I$ and $J$ bands. The contribution from the nebular continuum, which begins to be important at these wavelengths, has been estimated at less than or around $10 \%$ using the standard techniques presented in Osterbrock (1989).

It is seen in Table 2 that the colors of the inner zones are usually bluer than those of the outer zones of the line-emitting region, in accordance with the Guzmán et al. (1998) color profiles. This can be interpreted in light of the results presented in $\S 3.2$. Whenever one region in a galaxy is redder than another region, it can be caused by differences in reddening, metallicity, or age. The first of these factors is unlikely to play a role in this particular case, since the reddening has been estimated, and it is very small. The second factor is also unlikely to be responsible for the observed color gradients, since the metallicity differences are likely to be very small in these systems at such short spatial scales because of very efficient mixing by supernova-driven winds.
Moreover, we conclude that the observed color differences between the $\mathrm{a}$ and $\mathrm{b}$ zones are more likely to arise from age differences between these two zones. Specifically, the underlying stellar populations in the $b$ zones are typically older than the stellar populations of the a zones of their respective galaxies. The chemical enrichment histories of both regions have probably been very similar for the reasons outlined above. This is an important finding for these distant and compact sources.

Alternatively, it might be possible to think that the color gradient is caused by mass segregation. If, for instance, most of the newly formed ionizing stars were in the a zone, the color of the inner part would be naturally bluer than the color of the outer zone. This scenario could also explain the observed differences in the ionization ratio between the two zones, which would be higher in the inner zone because of the higher amount of ionizing photons available to ionize the inner parts of the nebula. Although mass segregation is a concern for massive clusters (see, e.g., Stolte et al. 2005 and de Grijs et al. 2002), it only operates at very short distances (10 pc at most), and not at the much larger scales of interest in the present case. The possibility of a spatially varying present-day mass function can then be safely ruled out.

\section{THE EVOLUTIONARY MODELS}

The observational data gathered for the LCBGs presented here can be used to construct galaxy models that represent the objects. Although the models presented are very crude and tailored, they provide at least an idea of the stellar inventory and possible evolution of these LCBGs. This will shed light on the possible evolution and age of these galaxies. In this section, the star formation history of the four LCBGs is studied using evolutionary population synthesis techniques. The models have been built using the Bruzual \& Charlot (2003) and Leitherer et al. (1999) evolutionary models. For each galaxy and extraction, a or b, two simple stellar populations are combined. One population is designed to mimic the observed properties of the ionizing stellar population, and the other is chosen to match the observed colors when combined with the ionizing population. This latter population therefore represents the underlying population. We note that the population analysis used here is carried out for both the inner and outer zones of the line-emitting region separately, taking advantage of the HST spatial resolution. In addition, the fact that the star-forming region is split into two subregions can also help to model possible delays between the star formation episodes in the inner zone and in the outer zone of the line-emitting region.

The metal content of the ionizing stellar population is assumed to be equal to the metal content of the ionized gas. Since the only information available on the metal content of the ionizing population of these sources comes from the gas-phase oxygen abundances, ${ }^{12}$ two sets of models are constructed. Model set 1 assumes that the ionizing population has a metallicity of $Z=$ 0.008 . Model set 2 adopts $Z=0.004$ for the metal content of the ionizing population. These two numbers can be mapped into $12+\log (\mathrm{O} / \mathrm{H})=8.4$ and 8.0, approximately, assuming solar chemical proportions. Therefore, for each galaxy and extraction, two models of different metallicities are built. The stellar inventory of the observed galaxies is described according the following steps:

1. The first step is the derivation of the masses of the ionizing populations.

\footnotetext{
12 The value of $12+\log (\mathrm{O} / \mathrm{H})$ lies between 7.8 and 8.4 , although the preferred value is 8.4 because of the Guzmán et al. (1996) HIRES measurements.
} 
2. The second step is the determination of the ages and colors of the ionizing populations. In this step the Leitherer et al. (1999) models are used.

3 . In the third step the mass of the underlying stellar population is derived, together with its age. The Bruzual \& Charlot (2003) models are used to represent the older stellar generation.

4. The last step is a double-check of the derived masses using the measured NICMOS luminosities.

\subsection{The Mass of the Ionizing Population}

The mass of the ionizing population is a very important parameter that defines it. If the mass of the ionizing population is high enough, the initial mass function (IMF) will be well sampled. If the IMF is not well sampled, it might be the case that the models cannot be used to describe the stellar population. This effect is commonly known as the "richness effect," or the "Poisson shot noise." Depending on the slope and cutoff masses of the IMF used, this can become a problem for population masses lower than $10^{5} M_{\odot}$, but for the usual Salpeter IMF the mass limit is lower. This issue has been extensively studied in the series of papers by Cerviño et al. $(2000,2001,2002)$.

The mass of the ionizing population, $M_{*}$, can, in principle, be derived from the luminosities of the observed recombination lines according to the following considerations. As the stellar population ages, the number of hydrogen-ionizing photons per unit mass of the ionizing population also decreases. Assuming that the age of the ionizing population is related to the intrinsic $W_{\mathrm{H} \beta}$, a relation should exist between the number of hydrogen-ionizing photons per unit mass of the single stellar generation and $W_{\beta}$. Such a relation is given for single-burst models in Díaz et al. (2000b). The expression used is

$$
\log \left[Q(\mathrm{H}) / M_{*}\right]=44.8+0.86 \log W_{\mathrm{H} \beta},
$$

where $Q(\mathrm{H})$ is the number of hydrogen-ionizing photons per second, $M_{*}$ is expressed in solar masses, and $W_{\mathrm{H} \beta}$ is the "intrinsic" equivalent width, the one that would be observed if no underlying stellar population were present. Again, this expression works best in stellar populations with well-sampled IMFs. The number of ionizing photons is an integral over the mass range more affected by the richness effect, and it is therefore very sensitive to small number statistics when applied to low-mass populations. The IMF used to derive this expression had an exponent of 2.35 , a lower mass limit of $0.85 M_{\odot}$, and an upper mass limit of $120 M \odot$.

However, the presence of the underlying stellar population introduces a degeneracy in the measurements of the equivalent widths. This prevents us from measuring directly the equivalent widths that would be observed if only the ionizing stellar population were present. The main goal of the stellar population analysis presented here is then to unravel the different stellar populations. This is carried out following the ideas presented in Díaz et al. (2000a). In order to solve this degeneracy in equivalent width introduced by the presence of the underlying population, the upper envelope of the relationship between $\log W_{\mathrm{H} \beta}$ and $\log \left(\left[\mathrm{O}_{\mathrm{III}}\right] /\right.$ [O II]) presented in Hoyos \& Díaz (2006, Fig. 6) was used to estimate the equivalent width that would be observed in the absence of a previous stellar generation. This "effective" equivalent width is called $W_{\beta}^{0}$. This assumption is now briefly justified. In the $W_{\mathrm{H} \beta}$-to-[O $\left.\mathrm{OIII}\right] \lambda \lambda 4959+5007 /[\mathrm{O}$ II] $] \lambda 3727$ ratio diagram for very luminous $\mathrm{H}$ II galaxies, if the observed range of ionization degrees is ascribed to the age of the ionizing population, it is reasonable to assume that the vertical scatter shown by the data is caused by different contributions of continuum light from the underlying population. ${ }^{13}$ The expression used is

$$
\log W_{\mathrm{eff}, \beta}^{0}=2.00 \pm 0.02+(0.703 \pm 0.028) \log [\mathrm{O} \mathrm{III}] /[\mathrm{O} \text { II] } .
$$

The upper envelope of this relation, which defines the "effective" equivalent width, is then relevant to estimate the "intrinsic" equivalent width because the objects located on top of this upper envelope are likely to be the ones in which the underlying population contribution is minimal. This effective equivalent width allows us to estimate the mass of the ionizing population assuming that $W_{\beta}^{0}$ is equal to the real, or intrinsic, equivalent width that would be measured if the starburst could be observed in isolation. This is done via the expression given above using the measured $\mathrm{H} \beta$ luminosities to obtain the number of hydrogen-ionizing photons per second through standard nebular analysis techniques (Osterbrock 1989). The resulting stellar masses of the ionizing population are given in Table 3 . Typical ionizing population masses range from 8 to 30 million $M_{\odot}$. The systematic errors affecting these calculations are explained in the following paragraphs.

The effective equivalent widths, which are mostly in the 100 $200 \AA$ interval, are still lower limits only to the real value of the equivalent width, because the empirical method used here to derive the former does not forbid the existence of objects with higher observed equivalent widths for their observed ionization ratio $\left[\mathrm{O}_{\text {III }}\right] /\left[\mathrm{O}_{\mathrm{II}}\right]$. This is indeed a possibility, since the work presented in Hoyos \& Díaz (2006) used local galaxies, and the galaxies analyzed here are intermediate- redshift sources. Therefore, the derived value for the mass of the ionizing populations obtained using $Q(\mathrm{H})$ (or, rather, the $\mathrm{H} \beta$ luminosity) and $W_{\beta}^{0}$ can only be lower limits to the real masses. It has to be said, however, that the possible differences between the intrinsic and the derived effective equivalent widths cannot be very high. The maximum attainable equivalent width, which corresponds to the case in which only the nebular continuum is considered, is $1000 \AA$. When the stellar continuum is taken into account, the maximum equivalent width drops to about $400 \AA$, depending on the IMF, metallicity, and age of the population, and the largest $\mathrm{H} \beta$ equivalent widths ever measured are close to this latter quantity. This implies that the intrinsic equivalent widths of the observed starbursts have to be in the $200-400 \AA$ range. These considerations allow us to estimate the possible systematic errors introduced by this approximation. Using the typical effective equivalent width and an average value for the maximum intrinsic equivalent width, the real masses of the ionizing populations could be up to a factor of 2 larger. However, even if the intrinsic value could be observed or somehow derived, the mass of the ionizing component obtained would always remain a lower limit because some photons could actually be escaping from the nebula, and dust globules will always be present amid the gas clouds. Moreover, if the ionizing population turned out to emit more photons harder than 1 ryd than the number predicted by the models used to derive equation (5), the mass would be underestimated, too. The new Geneva models with rotation at low metallicity (Maeder \& Meynet 2001) point in this direction. For these reasons, further steps

\footnotetext{
13 There is, however, a metallicity effect. In the absence of an underlying stellar population, $\mathrm{H}$ II galaxies with higher metallicity clouds will present a lower ionization ratio due to lower effective temperatures of their ionizing stars. However, there will be no change in the equivalent width of $\mathrm{H} \beta$, to zeroth order. Therefore, $W_{\beta}^{0}$ should be a function of both the ionization ratio and metallicity. Only the ionization ratio dependence of the "effective" equivalent width is used here.
} 
TABLE 3

Model Results

\begin{tabular}{|c|c|c|c|c|c|c|c|c|}
\hline \multirow[b]{2}{*}{ Parameter } & \multicolumn{2}{|c|}{ ОвЈест 1} & \multicolumn{2}{|c|}{ ОвЈест 2} & \multicolumn{2}{|c|}{ ОвЈест 3} & \multicolumn{2}{|c|}{ ОвЈест 4} \\
\hline & Inner & Outer & Inner & Outer & Inner & Outer & Inner & Outer \\
\hline \multicolumn{9}{|c|}{ Model Set 1} \\
\hline Age of the ionizing cluster $(\mathrm{Myr})$ & 3.1 & 3.2 & 4.8 & 4.9 & 3.2 & 3.2 & 3.3 & 3.3 \\
\hline$B-V$ color of the ionizing cluster & $-0.16 \pm 0.04$ & $-0.14 \pm 0.04$ & $-0.10 \pm 0.04$ & $-0.08 \pm 0.04$ & $-0.14 \pm 0.04$ & $-0.14 \pm 0.04$ & $-0.15 \pm 0.04$ & $-0.15 \pm 0.04$ \\
\hline Age of the underlying population (Myr) & $500 \pm 100$ & $1500 \pm 800$ & $900 \pm 200$ & $800 \pm 200$ & $18 \pm 5$ & $280 \pm 70$ & $400 \pm 100$ & $1300 \pm 600$ \\
\hline $\log M / M_{\odot}$ (ionizing cluster) & 6.9 & 7.11 & 6.5 & 6.7 & 6.8 & 7.0 & 7.1 & 7.2 \\
\hline 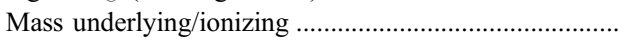 & $80 \pm 20$ & $140 \pm 30$ & $260 \pm 50$ & $1340 \pm 300$ & $9 \pm 2$ & $100 \pm 20$ & $80 \pm 20$ & $190 \pm 40$ \\
\hline 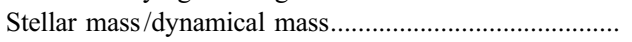 & 0.20 & $\ldots$ & 0.60 & $\ldots$ & 0.20 & $\ldots$ & 0.60 & $\ldots$ \\
\hline $\log M / M_{\odot}(\mathrm{STIS}+\mathrm{WFPC} 2) \ldots \ldots$ & 9.4 & $\ldots$ & 9.9 & $\ldots$ & 9.0 & $\ldots$ & 9.6 & $\ldots$ \\
\hline $\log M / M_{\odot}(\mathrm{NICMOS})$ & 9.5 & $\ldots$ & 9.7 & $\ldots$ & 9.1 & $\ldots$ & 9.7 & $\ldots$ \\
\hline 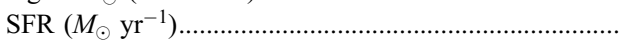 & $6.5 \pm 0.3$ & $\cdots$ & $0.8 \pm 0.06$ & $\ldots$ & $4.7 \pm 1.0$ & $\ldots$ & $6.8 \pm 1.2$ & $\ldots$ \\
\hline \multicolumn{9}{|c|}{ Model Set 2} \\
\hline Age of the ionizing cluster (Myr) & 2.9 & 3.2 & 4.9 & 5.1 & 2.9 & 4.0 & 4.0 & 4.1 \\
\hline 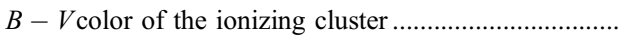 & $-0.17 \pm 0.04$ & $-0.14 \pm 0.04$ & $-0.08 \pm 0.04$ & $-0.07 \pm 0.04$ & $-0.17 \pm 0.04$ & $-0.12 \pm 0.04$ & $-0.13 \pm 0.04$ & $-0.13 \pm 0.04$ \\
\hline Age of the underlying population & $600 \pm 200$ & $790 \pm 200$ & $1000 \pm 500$ & $1000 \pm 250$ & $60 \pm 20$ & $550 \pm 100$ & $400 \pm 100$ & $900 \pm 200$ \\
\hline $\log M / M_{\odot}$ (ionizing cluster) & 6.9 & 7.11 & 6.5 & 6.7 & 6.8 & 7.0 & 7.1 & 7.2 \\
\hline 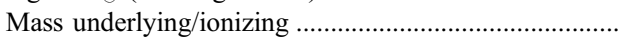 & $70 \pm 10$ & $90 \pm 20$ & $500 \pm 100$ & $800 \pm 200$ & $28 \pm 6$ & $220 \pm 40$ & $90 \pm 20$ & $250 \pm 50$ \\
\hline 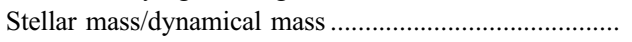 & 0.05 & $\ldots$ & 0.40 & $\ldots$ & 0.40 & $\ldots$ & 0.70 & $\ldots$ \\
\hline $\log M / M_{\odot}(\mathrm{STIS}+\mathrm{WFPC} 2) \ldots \ldots$ & 9.2 & $\ldots$ & 9.7 & $\ldots$ & 9.4 & $\ldots$ & 9.7 & $\ldots$ \\
\hline $\log M / M_{\odot}(\mathrm{NICMOS})$ & 9.6 & $\ldots$ & 9.9 & $\ldots$ & 9.5 & $\ldots$ & 10.0 & $\ldots$ \\
\hline $\operatorname{SFR}\left(M_{\odot} \mathrm{yr}^{-1}\right)$ & $6.5 \pm 0.3$ & $\ldots$ & $0.8 \pm 0.06$ & $\ldots$ & $4.7 \pm 1.0$ & $\ldots$ & $6.8 \pm 1.2$ & $\ldots$ \\
\hline
\end{tabular}

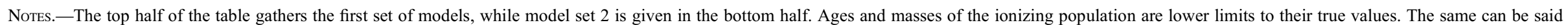

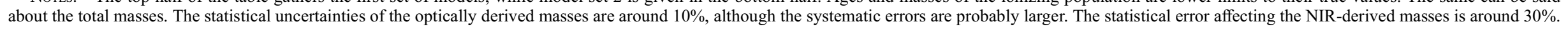




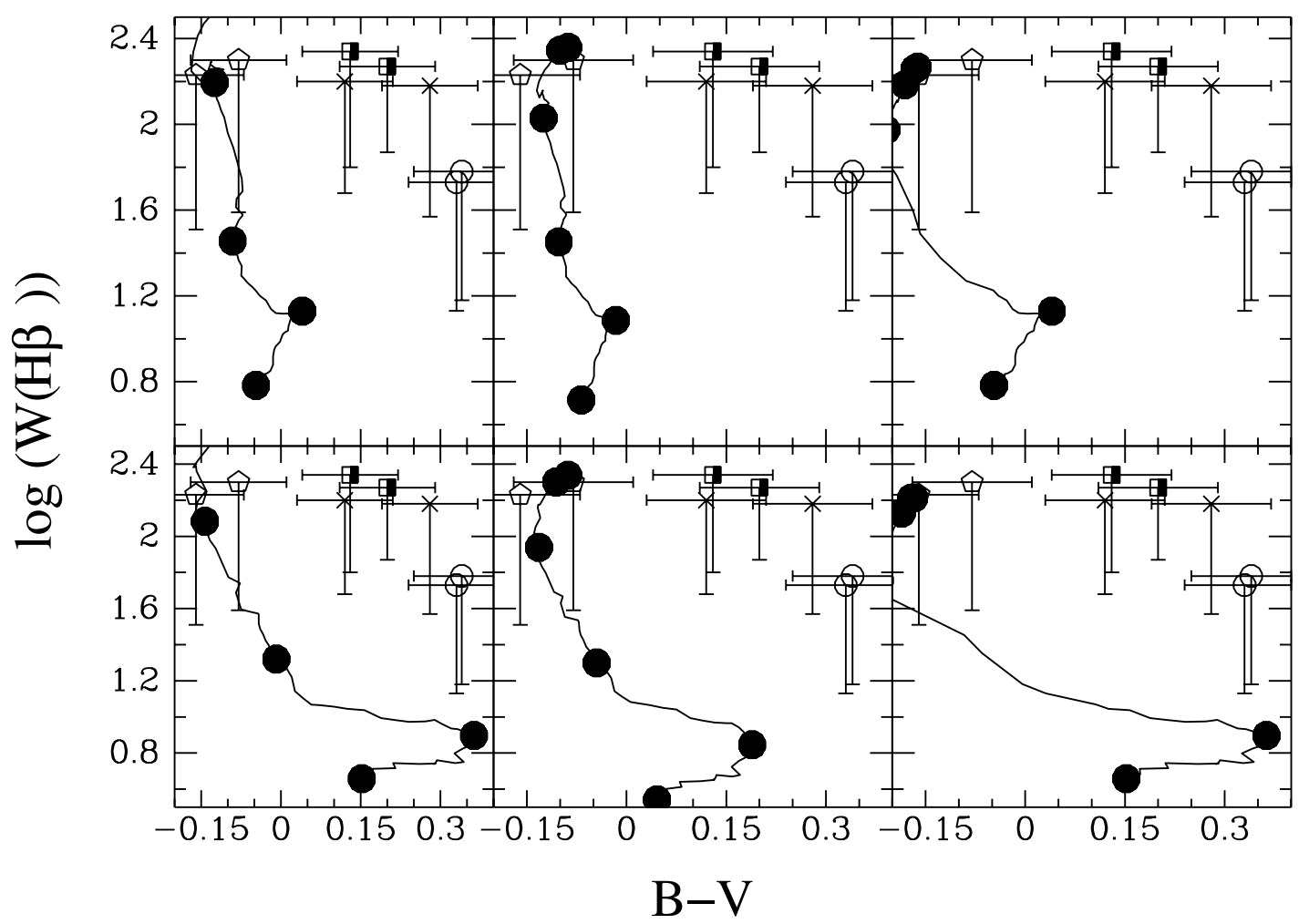

Fig. 4. - Evolution of a single stellar population in the $\log W_{\mathrm{H} \beta}-(B-V)$ plane from $t=0$ to $t=10$ Myr. The data were taken from the Leitherer et al. (1999) library. In all cases, an instantaneous burst is assumed, and the nebular continuum emission is taken into account. Two different oxygen contents $(Z=0.008$, model set 1 , bottom panels; $Z=0.004$, model set 2, top panels) and three different IMFs (Salpeter 1.0-100 $M_{\odot}$, left panels; power law with an exponent $\alpha=3.3$ with the same mass limits, middle panels; Salpeter IMF truncated at $30 M_{\odot}$, right panels) are plotted. For the LCBG points, $W_{\beta}^{0}$ is plotted against the emission-line-free, reddening-corrected color. The horizontal error bars represent the 0.09 mag uncertainty, and the vertical semierror bars reach to the observed equivalent width, in order to indicate the importance of the contribution of the underlying stellar population to the optical light. The filled circles represent the $0,2,4,6,8$, and $10 \times 10^{6} \mathrm{yr}$ checkpoints. Object 1 is represented by half-filled squares, object 2 is represented by open circles, object 3 is represented by pentagons, and crosses denote object 4 . Two symbols are shown for each object, corresponding to the $\mathrm{a}$ and $\mathrm{b}$ extractions. In all cases, the $\mathrm{b}$ apertures have redder colors.

toward the determination of the intrinsic equivalent width from the effective equivalent width were not taken, and both quantities are assumed to be equal.

\subsection{Determination of the Age and Intrinsic Color of the Ionizing Population}

The value of the effective equivalent width can be used to obtain more information about the underlying stellar populations via the Leitherer et al. (1999) models, making some assumptions about the metallicity and IMF of this stellar generation. Again, the effective equivalent width allows us to estimate, or at least to place significant lower limits on, the age and intrinsic color of the ionizing population, assuming that $W_{\beta}^{0}$ is equal to the real or intrinsic equivalent width that would be measured if the starburst could be observed in isolation. The Leitherer et al. (1999) models are used now.

Figure 4 presents nine models from the Starburst99 (Leitherer et al. 1999) library that represent the evolution in the $\log W_{\beta}$ versus $B-V$ plane of a single stellar population from its birth until it is $10 \mathrm{Myr}$ old. The nebular continuum emission is included in these diagrams. This evolution is shown by the solid lines in the figure. Ages increase from top to bottom. Three different metallicities and IMFs are presented, as indicated in the figure. The derived $W_{\beta}^{0}$ for both the inner and outer zones of the line-emitting regions of the observed sources is plotted against the emissionline-free, reddening-corrected color in the case of the studied LCBGs. This is the color that would be measured if the stars could be observed in isolation.
It is interesting to note that the values of the effective equivalent width are close to the maximum equivalent width value of the plotted curves, which is the maximum value of the intrinsic equivalent width for zero-age clusters of the corresponding metallicity and IMF,${ }^{14}$ further highlighting the fact that, even though the effective equivalent width is a lower limit to the real value, it is a reasonable approximation to it, especially if one considers that, even if the real value could be somehow known, it would still yield lower limits to the mass of the ionizing clusters because of photon escape and dust issues. It is clearly seen that threefourths of the regions studied require the presence of an underlying stellar population, since they do not lie along a solid line. Therefore, they cannot be reproduced by a single stellar population. It is also seen that the single stellar populations with a truncated Salpeter IMF have maximum intrinsic equivalent widths which are lower than the derived values of $W_{\beta}^{0}$ for many regions. This means that we can rule out these models as descriptions of the ionizing stellar populations found in the observed LCBGs, since the effective equivalent widths are in any case only lower limits to the real ones. This can be interpreted in the sense that the observed galaxies have ionizing populations so massive that they fully sample their respective IMFs, which is a necessary condition to achieve high equivalent widths. In addition, the models whose IMFs have a slope of 3.33 are redder than the inner region of object 3, which makes it impossible to accommodate an older and redder underlying population in this model, and there are

\footnotetext{
${ }^{14}$ About $350 \AA$ for the presented cases.
} 
also some regions with $W_{\beta}^{0}$ greater than the zero-age equivalent width of those models. The models with Salpeter IMFs are therefore chosen to describe the ionizing populations, simply because they are compatible with all these restrictions. Although the reasons to discard the $m^{-3.33}$ IMF are of course weaker, considering multiple IMFs is by no means justified given the few input data at our disposal. The use of the Salpeter IMF provides a crude yet consistent description of the ionizing population found in the observed regions.

Assuming that $W_{\beta}^{0}$ equals the real equivalent width, as was done before, and using the Salpeter IMF for the two metallicities that correspond to the metallicities of the two model sets used, it is possible to calculate the ages and intrinsic colors of the ionizing population. They are given in Table 3 . The ionizing clusters are found to be very young (less than 5 Myr in most cases). As before, the use of the effective equivalent width instead of the unknown intrinsic value introduces a systematic error in the age determinations. The ionizing populations are then bound to be even younger than the presented quantities. When dealing with very young coeval single stellar populations, it has to be kept in mind that the observed properties of such populations do not change to a great extent until they are about 3 Myr old, simply because of the fact that this is the minimum lifetime of even the most massive stars, and also because the approximation of an instantaneous burst might fail at such short timescales. The main conclusion is that the ionizing populations of the observed regions are probably extremely young. The systematic error in the intrinsic color of the ionizing population is also estimated from the color scatter of the models used between zero age and the maximum age allowed by the observed $W_{\beta}^{0}$. All errors are 0.04 mag.

\subsection{The Age and Mass of the Underlying Population}

The next step in describing the stellar populations found in the observed LCBGs is calculating the age and initial mass of the underlying stellar population. The single stellar populations used to describe the underlying generation of stars are chosen from the Bruzual \& Charlot (2003) library. The evolutionary tracks used are the 1994 Padova ones, and the IMF chosen is the Salpeter IMF. The metallicity of this underlying stellar population is $Z=0.004$ for the case of model set 1 and $Z=0.0004$ for the case of model set 2 . These are lower than the metallicities used to describe the ionizing populations. In this step, the ionizing cluster is represented by the single stellar population available in the Bruzual \& Charlot (2003) library that most closely resembles the Leitherer et al. (1999) model used before in terms of metallicity, IMF, and $W_{\beta}^{0}$-derived age.

Assuming that the metallicities of the ionizing populations are higher than the metallicities of the underlying populations tries to emulate the chemical evolution of the observed galaxies from their inception until the onset of the observed starbursts. Although the choice adopted is not justified by the use of any particular chemical evolution model, it is at least a zeroth-order solution to the problem, since the Bruzual \& Charlot (2003) models only offer a limited array of metallicities for the different single stellar populations. In the framework of the closed-box model and the instantaneous recycling approximation, the corresponding gas consumption fractions for the required metal enrichments are $10 \%$ for model set 1 and $60 \%$ for model set 2 , assuming zero metallicity in the distant past, before the starbursts that gave birth to the underlying stellar generations took place. The gas consumption fraction is larger in the metal-poor models, since the difference in metallicity between the two simple populations mixed to construct them $\left(Z_{\text {ion }}=0.004, Z_{\text {under }}=0.0004\right)$ is much larger than the metallicity difference of the two simple populations combined for the first models $\left(Z_{\text {ion }}=0.008, Z_{\text {under }}=0.004\right)$. The yields provided by M. Mollá (2006, private communication) and Maeder (1992) were used in these latter calculations. Although these estimates, by themselves, do not prove that this picture is accurate to describe the star formation history of these objects, they show that the assumed metallicity differences between the stellar populations used to build the models are at least possible. In addition, it has to be kept in mind that the global remaining gas fraction is bound to be lower for the metal-rich models in the framework of the closed-box model and the instantaneous recycling approximation than for the metal-poor models. In particular, given the assumed metallicity for the ionizing population of the second set of models, the gas consumption fractions of pristine gas have to be higher than $60 \%$ in both cases, again in the closed-box model with the instantaneous recycling approximation.

The age and mass of the second stellar population are then chosen so that the predicted equivalent width by the time the current starburst is observed matches the measured equivalent width and the total color equals the calculated rest-frame, stellarpopulation-only, extinction-corrected $B-V$ color. In essence, this procedure estimates the amount of "red light" that has to be added to the "blue light" of the ionizing population in order to match the two observables available $(B-V$ color and measured $\mathrm{H} \beta$ equivalent width) at the time in which the observed starburst takes place. This second stellar population is therefore designed to match the older stellar population.

The difference between the observed $B-V$ color and the previously derived intrinsic $B-V$ color of the ionizing population (given in Table 3) can be approximately matched with "how red," and the difference between the observed equivalent width and the intrinsic equivalent width roughly translates into "how much" red light has to be added, although both quantities are of course interlaced.

The errors in the observed equivalent widths (typically 17\%) and stellar colors ( $0.09 \mathrm{mag}$, as estimated above) translate themselves into errors in the relative mass of the underlying stellar population with respect to the ionizing population and age. Although it is not true that $100 \%$ of the error in the relative mass comes from the uncertainties in the measured equivalent width and that $100 \%$ of the error in the age of the underlying stellar population arises from the errors in the derived stellar colors, ${ }^{15}$ these are the main dependencies. Assuming that this simplified error scheme holds, the typical uncertainties in the relative mass of the ionizing cluster with respect to the mass of the ionizing cluster are $20 \%$. The uncertainties in the age of the underlying population are $25 \%$ for ages below $10^{9} \mathrm{yr}$ and twice this amount for older ages. This is because of the flattening in the $B-V$-age relationship of the Bruzual \& Charlot (2003) models. The results are also presented in Table 3.

\subsection{NICMOS NIR Photometric Masses}

The final step in this crude description of the stellar populations found in the observed LCBGs is made possible by the use of NIR data. This information is now employed to check the likelihood of the derived stellar populations by using the total luminosities emerging from the line-emitting regions in the wavelength range that transforms into the F160W wavelength interval measured by NICMOS to rederive the total stellar masses contained in the line-emitting region, which is the area probed by the STIS spectra. This is done by calculating the relevant $M / L_{X}$ ratios via

\footnotetext{
15 In the sense that, for instance, isoage contours in the age-relative mass plane do not map directly into iso- $W_{\beta}$ contours in the $W_{\beta}$-stellar color plane.
} 
the $M / L_{B}{ }^{16}$ ratios, making use of the programs presented in Bruzual \& Charlot (2003). The statistical uncertainty that affects these NIR-derived photometric masses is a combination of two effects. The first effect is the intrinsic uncertainty in the flux measurement, and the second effect comes from the scatter in the $L_{B} / L_{X}$ ratio. This latter error source dominates the first one, having a scatter of $30 \%$. This number was derived by recalculating the $L_{B} / L_{X}$ number for different Bruzual \& Charlot spectra of the two metallicities considered for the underlying stellar populations, with the typical ages predicted in the models previously constructed.

Total model masses, NIR photometric masses, and the ratio of the two are given in Table 3. It is seen in the table that the first set of models produces NIR photometric masses that are in very good agreement (to within 25\%) with the masses derived using only the spectroscopic and optical photometry. The agreement for the second set of models is somewhat worse (to within a factor of 2). These results lead us to think that model set 1 is a better overall description.

\subsection{Mental Model for the Observed Objects}

Table 3 lists the ages and masses of the stellar populations that best represent the observed objects in the framework of the presented models. This table also gathers the SFRs and the percentage of the dynamical mass that is accounted for by the stellar populations used.

The SFR was calculated from the $\mathrm{H} \alpha$ luminosity as in Kennicutt et al. (1994), valid for $T_{e}=10^{4} \mathrm{~K}$ and case B recombination (all the ionizing photons are processed by the nebular gas). The results are given in Table 3.

$$
\mathrm{SFR} M_{\odot} \mathrm{yr}^{-1}=7.9 \times 10^{-42} L_{\mathrm{H} \alpha} \operatorname{ergs~s}^{-1}
$$

Although the preceding SFR estimate was based on spatially averaged SFRs for disk galaxies, we believe it can be applied to the current sample of starburst galaxies. The $\mathrm{H} \alpha$-derived SFRs only probe the most recent star formation episodes of any given galaxy because the $\mathrm{H} \alpha$ line becomes very faint soon after the more massive stars, born in the more massive starbursts, die out, and this is true for all normal galaxies. For this reason, it is possible to use the Kennicutt et al. (1994) expression for the global SFR of actively star-forming galaxies, too. In fact, the SFR $\mathrm{H} \alpha$ estimate has been calibrated against many other instantaneous star formation tracers (Rosa-González et al. 2002). Differences between the Kennicutt et al. (1994) H $\alpha$-derived SFR and the real SFRs of the studied galaxies are bound to arise mostly from other issues, such as differences in the IMFs used.

The calculated SFRs are in the range from 0.8 to $6.8 M_{\odot} \mathrm{yr}^{-1}$, indicating that the star-forming episode is very strong (the 30 Doradus SFR is $0.1 M_{\odot} \mathrm{yr}^{-1}$ ). However, it has to be said that the line-emitting regions typically span around $3 \mathrm{kpc}$, and the 30 Doradus line-emitting region is a only few hundred parsecs. The SFRs per unit area are therefore of the same order of magnitude.

Table 3 shows that the ionizing populations are very young, less than $5 \mathrm{Myr}$ in almost all cases, although the average age is slightly over 3 Myr. The ionizing clusters are then largely unevolved. The resulting ages in both model sets indicate that these galaxies are being observed at the peak (or a little bit past the peak) of their line luminosities. It is also the case that the ages of

\footnotetext{
16 The $L_{B} / L_{X}$ ratios are calculated for the (previously derived ) model that best describes each particular object. The two families of model set 1 and model set 2 are used.
}

the ionizing populations are very similar for the inner and outer regions of each galaxy.

The inferred underlying populations are very different among the studied galaxies. There are systems with fairly young nonionizing populations (around $300 \mathrm{Myr}$ old), but there are LCBGs whose underlying populations are very old (older than $1.0 \mathrm{Gyr}$ ). In the case of the inner region of SA57-10601, the estimated stellar population is compatible with a single ionizing stellar population. The modeled age of the older stellar population is usually larger for the outer regions than for the inner regions of each object. However, given the uncertainties in the age estimates for the underlying populations (between $25 \%$ and $50 \%$ ), it is not possible to extract much information from these ages alone. The average age of the underlying stellar populations found is $700 \mathrm{Myr}$ for both model set 1 and model set 2 .

The derived stellar masses account for $20 \%-60 \%$ (the average value being $40 \%$ ) of the virial masses within $R_{e}$, according to the first set of models. In the case of model set 2, the stellar mass accounts for $4 \%-70 \%$ (the average value is again $40 \%$ ) of the derived virial masses, where the virial masses were determined as in Hoyos et al. (2004), assuming that the measured gas-phase velocity dispersion equals the star's velocity dispersion and that LCBGs are in dynamical equilibrium. These are the virial masses within $R_{e}$. Since the mass estimates yielded by the models used here are only lower limits for the reasons outlined above, ${ }^{17}$ there is not much room left for the presence of dark matter in the innermost regions of these objects. The dark matter would, in any case, be spread out in a vast halo around the objects by its very nature. The presence or not of significant amounts of dark matter in the optical cores of these systems is important. If the conjecture introduced here that these systems are not dominated by dark matter in their very centers turns out to be true, it would imply that these systems could not possibly evolve into today's population of low surface brightness objects. A very simple and crude estimate based on the Mamon \& Lokas (2005) models and the Navarro et al. (2004) simulations indicates that the mass of dark matter within a $1.5 \mathrm{kpc}$ sphere ${ }^{18}$ is $\sim 2 \times 10^{8} \pm 40 \%$, assuming that the total mass of the dark matter halo is 10 times the measured kinematic mass. This contribution is much less than the kinematic masses derived from velocity dispersions. In order to determine the dark matter content and density profile of these sources with greater accuracy, measurements of the line-of-sight velocity distribution out to several (around 5-9) effective radii would be required. Such data are, of course, not available for the distant objects studied here.

It might be tempting to think that the remaining $60 \%$ of the dynamical mass not accounted for by the stellar component might be hinting at the existence of a very old stellar population, too faint and red to leave a trace in the $B-V$ color used here but as massive as the already detected underlying stellar population. However, this hypothetical phantom population cannot be older than $\sim 5 \mathrm{Gyr}$, simply because of look-back time, implying that such a very old and massive population cannot possibly exist. Such a population would still be shining in the $B-V$ color $^{19}$ because the turnoff point of this population would be around solar-type stars or slightly earlier. More strongly, the good agreement between the optically derived masses and the NIR-derived masses sets very strict limits

\footnotetext{
17 We remind the reader that the masses of the underlying populations are scaled with respect to the mass of the ionizing population. This is calculated via $W_{\beta}^{0}$, which is in turn a lower limit to the real equivalent width. Other factors such as photon escape, dust, and enhanced photon production have an impact, too.

18 Similar in size to $R_{e}$.

${ }^{19}$ Assuming that these stars occupy a volume similar to the other populations; i.e., the surface brightness of this population is not lowered for other reasons.
} 
on the luminosity of this possible population, virtually forbidding it for the case of model set 1 and for some of model set 2 as well. Finally, the presence of significant amounts of neutral or molecular gas in the central regions of the sources studied can be safely discarded. It is very likely that the extreme starburst taking place at the cores of these objects is well capable of fully ionizing the ISM in the innermost zones of these galaxies.

It is therefore concluded that the stellar inventory found using the simple models devised here cannot be very far from reality and that the disagreement between the stellar masses and the dynamical masses arises from the fact that the mass calculations for the stellar component are forcibly lower limits.

The models constructed here are similar to the two-burst models presented in Guzmán et al. (1998). In the models presented here, the ionizing cluster is less massive than the ionizing clusters used in that work. In addition, the underlying stellar populations used in Guzmán et al. (1998) are much older than the ones derived using the models presented here. The relative masses of the ionizing populations considered in Guzmán et al. (1998) went from $1 \%$ to $10 \%$, while the results presented in this paper lead toward much lower relative masses for the newer stellar generation, between $0.1 \%$ and $3 \%$. Another very important difference is that the underlying stellar population used in Guzmán et al. (1998) was set to be 3 Gyr old at the time of the ionizing generation birth, whereas in the current work this age is found to be much shorter, around $1 \mathrm{Gyr}$.

Finally, we close this section with a brief summary of how the models are built. This procedure is carried out for each region (a and b) and the assumed metallicity of each object. See Díaz et al. (2000a) for a complete account of how to mix several single stellar populations to match the observed properties of star-forming regions.

1. The first step is to derive the $\mathrm{H} \beta$ "effective" equivalent width. This is done via the ionization ratio $[\mathrm{O} \mathrm{III}] /[\mathrm{O} \mathrm{II}]$ and the relationship given above.

2. This "effective" equivalent is assumed to be equal to the "intrinsic" value and used to determine the age and $B-V$ color of the ionizing stellar population. In this step the error budget is dominated by the systematic error of assuming that both quantities are equal.

3. Using the measured $\mathrm{H} \beta$ luminosity, the mass of the ionizing population is estimated. This estimate can only be a lower limit to the real value because some unprocessed photons might be escaping from the nebula, and dust globules will be present. This also translates into the derived masses for the underlying stellar populations.

4. Using the emission-line-free $B-V$ color and the observed $\mathrm{H} \beta$ equivalent width, the age and relative mass of the nonionizing population are derived. The errors in these quantities are dominated by the uncertainties in the required color and required specific intensity at $4860 \AA$ of this population.

5. The NIR observations are then used to check whether the models created to describe the optical properties of the observed galaxies also describe their NIR properties. It is the case that the agreement is excellent for the metal-rich models, and somewhat worse for the metal-poor ones.

\section{COMPARISON WITH LOCAL GALAXIES: BRIGHT, LOCAL H II GALAXIES AS NEARBY LCBGs AND THE LCBG-SPHEROIDAL CONNECTION}

It is interesting to compare the evolution of the models described here with present-day galaxy samples. In this section several predicted observables are compared to the properties found in local systems. In particular, the issue of whether the stellar populations predicted by the presented models are similar to the underlying stellar populations of local $\mathrm{H}$ II galaxies or the less luminous BCDGs is addressed. This section also deals with the passive evolution of LCBGs and their connection with the local population of dwarf elliptical systems.

The H II galaxy sample used here was taken from the Telles \& Terlevich (1997) sample. This sample comprises 15 galaxies, which were selected from the Spectrophotometric Catalogue of H II Galaxies (Terlevich et al. 1991). Their equivalent widths are between 26 and $170 \AA$, and their $M_{B}$ values range between -14.5 and -21.5 , although with a preference for the brighter values. The measured velocity dispersions range from 16 to $50 \mathrm{~km} \mathrm{~s}^{-1}$. The galaxies also exhibit wide morphological variety. This sample was chosen because $\mathrm{H}$ II galaxies also show conspicuous and galaxy-wide emission-line spectra. They are quite similar to the LCBGs presented here in this respect, as hinted by Koo et al. (1994), Guzmán et al. (1997), and Hoyos et al. (2004). Their small radii and velocity dispersions indicate that they ought to be as massive as distant LCBGs. In addition, they are also known to possess violent star-forming systems, just like intermediate- $z$ LCBGs. It is then natural to wonder whether bright, local H II galaxies are nearby copies of distant LCBGs or whether they are different from the distant sources in some respect.

The BCDG control sample is the one found in Doublier et al. (1997, 1999). This sample consists of 44 galaxies, which were selected from the Second Byurakan Survey (Markarian 1967) and other lists. The Caon et al. (2005) BCDG sample was also included. It adds seven objects. Their luminosity profiles are typically $r^{1 / 4}$ profiles, although there is a sizable fraction of purely exponential profiles and mixtures of both profiles. Their star-forming regions are clearly detected in $B-R$ color maps and show an age spread. The presence of very old red giant stars indicates that these systems began to form their stars several gigayears ago, at the time in which the intermediate-redshift LCBGs were observed. This sample was chosen because BCDGs can be considered to be the low-luminosity end of $\mathrm{H}$ II galaxies in many respects. Therefore, they can be used to make educated guesses about the properties of other populations of faint intermediate-redshift objects whose luminosities are not high enough to be considered LCBGs and are therefore very difficult to study observationally, given their distances.

It is also possible to investigate the passive evolution of these systems in the framework of the presented models. This scenario of a passive evolution assumes that no further episodes of star formation take place from their look-back times to the present day. Given the high intensity of the observed starburst, it is not unlikely that the interstellar medium will be cast out of the galaxy, preventing further starbursts from happening. However, it has to be borne in mind that the look-back times of the LCBG sample used here are $\sim 5 \times 10^{9} \mathrm{yr}$. This means that there is a huge amount of time for them to undergo other star formation episodes, mergers, or other phenomena that would, of course, not be represented by the simple passive-evolution scenario used. In particular, the evolution of the intermediate-redshift LCBGs studied here into today's dwarf elliptical systems is studied. The dE/Sph sample used for comparison purposes is that of Guzmán (1994). We also use four other well-known dwarf elliptical galaxies from the local universe. The observed LCBGs are also compared with the LMC.

It is important to note that the available colors and magnitudes for the comparison samples are integrated data, while the models for the LCBG sample have been constructed from the line ratios and colors of the line-emitting region of each object. The model 

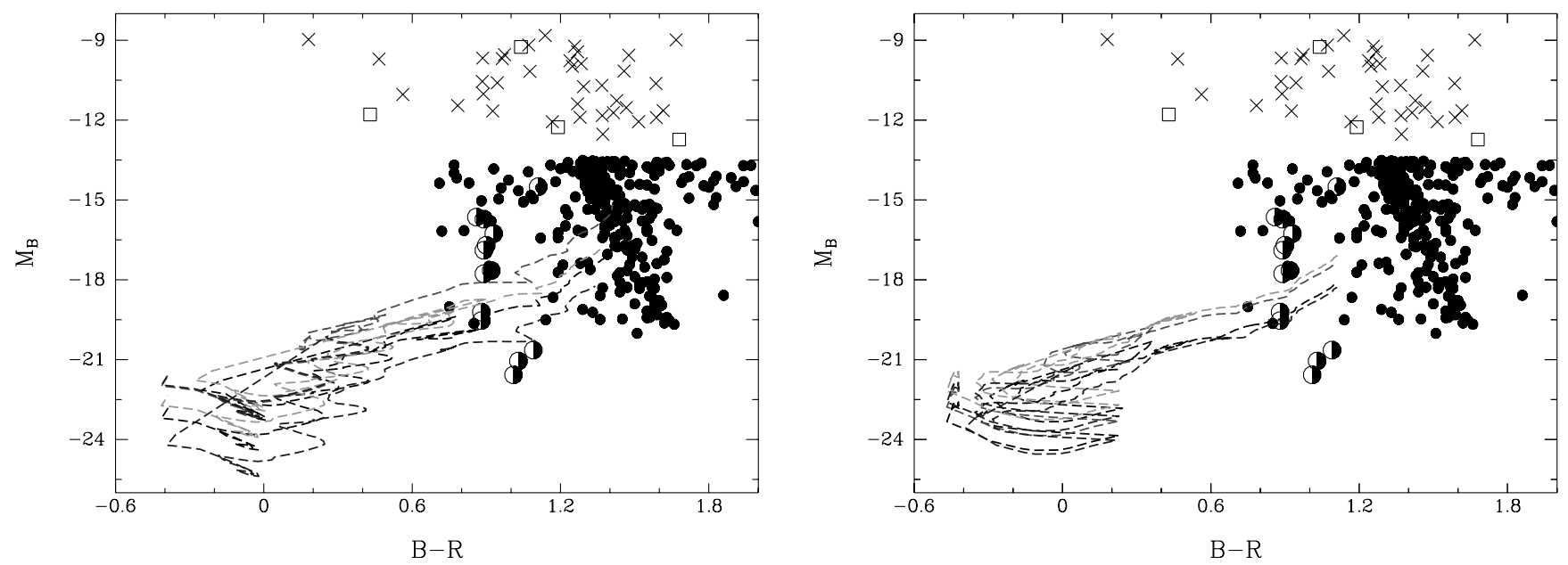

Fig. 5.- Model evolution in the $(B-R)-M_{B}$ plane. The light-gray line represents the evolution of the SA57-7042 models, the lower black line represents SA575482 , the dark-gray line represents the evolution of SA57-10601, and the upper black line represents H1-13088. The filled circles represent the dwarf elliptical systems from Guzmán (1994). Half-filled circles represent local H II galaxies from Telles \& Terlevich (1997). Crosses and squares represent the local BCDG samples from Doublier et al. (1997, 1999) and Caon et al. (2005), respectively. The left panel depicts the metal-rich models, whereas the right panel depicts model set 2. [See the electronic edition of the Journal for a color version of this figure.]

results for the inner and outer regions of each galaxy have therefore been mixed together and scaled using the appropriate weights to derive the properties of the whole sources. The main assumption is that the underlying stellar population of the central kiloparsec of these objects is qualitatively similar to the stellar population found in the outskirts ${ }^{20}$ of each galaxy. The absolute luminosities were further corrected by multiplying them by a factor of 2 to account for the luminosity of the stars found in the outskirts of the studied objects. ${ }^{21}$ This way, the observed galaxies can be represented by a single point each in color-color or color-magnitude diagrams.

Figure 5 presents the evolution of the stellar populations described by the models used here from their predicted creation time to the present day in the $M_{B}-(B-R)$ plane for the two sets of models used. The $B-R$ color information is an output from the Bruzual \& Charlot (2003) programs. The $B$ magnitude is the standard Johnson magnitude, while the $R$ magnitude is the Cousins $R$ magnitude. Each intermediate-redshift LCBG is represented by a line resulting from appropriately combining and scaling the models for the inner and outer zones of the line-emitting region, as stated above. This is also true for the rest of the diagrams. The Sph sample is represented by filled circles, and the local $\mathrm{H}$ II galaxies are represented by half-filled circles. The BCDGs from the Doublier et al. $(1997,1999)$ samples are depicted as crosses. The squares represent the Caon et al. (2005) galaxies.

The $B-R$ colors plotted in Figure 5 for the $\mathrm{H}$ II galaxies and BCDGs are not the $B-R$ colors of the whole galaxies; rather, they are the $B-R$ color of the underlying stellar component, excluding the starburst. This $B-R$ color has been estimated as the terminal color in $B-R$ color profiles, or from the $V-R$ colors of the extensions, assuming $B-V=0.65$. The $B-R$ color of the $\mathrm{dE}$ sample is the color of the entire galaxy, since there is no ionizing population in these objects. Therefore, it is possible to compare both $B-R$ color estimates in the sense that, if the $B-R$ color of the wings of an $\mathrm{H}$ II galaxy or BCDG is similar to the color of a Sph system, it would be possible to think that the starbursting H II galaxy or BCDG is a Sph system experiencing one further starburst. Figure 5 shows that the underlying stellar

\footnotetext{
${ }^{20}$ Here the word "outskirts" means "outside the line-emitting region."

21 This factor is needed because the STIS spectra only sample within $R_{e}$.
}

population of $\mathrm{H}$ II galaxies and most BCDGs are younger than the average $B-R$ color of Sph systems, implying that there are other differences between these galaxy types. In the case of the $\mathrm{H}$ II galaxies and BCDGs, the plotted values of $M_{B}$ are the blue absolute magnitudes of the stellar component only. These values were derived from the global $M_{B}$ using the appropriate available data in the references used and were corrected for galactic extinction according to Burstein \& Heiles (1982). The values of $M_{B}$ for the LCBG models were normalized to the starburst's luminosities, which are common to both the metal-rich and metal-poor models. The distance modulus used to derive the blue absolute magnitudes of the Guzmán (1994) sample from their reported apparent magnitudes is $35.0 \pm 0.3$, which is the average value of the Terlevich et al. (2001) sample. ${ }^{22}$ These transformations ensure that, when comparing all these samples with the LCBG models at $z=0$, colors and magnitudes are free from the contribution of emission lines, and the subsequent comparisons are between purely stellar properties only.

It can be seen in Figure 5 that the first set of models ends its evolution much closer to the bulk of the Sph systems than the second set of models. In fact, should the metallicity of LCBGs be on the lower end of the range allowed by their line ratios, the simple passive path assumed here could not transform them into Sph galaxies. To illustrate this point, a third, $10 \mathrm{Gyr}$ old stellar population was added to model set 2 . The mass of this population was arbitrarily set to $50 \%$ of the dynamical mass. Two different metal contents were tried for this primeval population, $Z=0.0004$ and 0.0001 , since higher metallicities are not justifiable in the framework of these metal-poor models. This population turned out to be unable to change the final $B-R$ color to a great extent, affecting it only on a scale of $0.05 \mathrm{mag}$. This possibility was not explored further. It is concluded that, if these intermediate-redshift LCBGs are to become Sph galaxies following a simple passive evolution, the first set of models is likely to be a better approximation to their stellar inventory. In order to reach more robust conclusions on the metallicity of LCBGs and their descendants, it would be necessary to compare the metallicity of LCBGs with that of Sph galaxies. However, the metal content of Sph galaxies is very difficult to determine spectroscopically

\footnotetext{
${ }^{22}$ Both studies dealt with the Coma Cluster, which is located at $100 \mathrm{Mpc}$.
} 

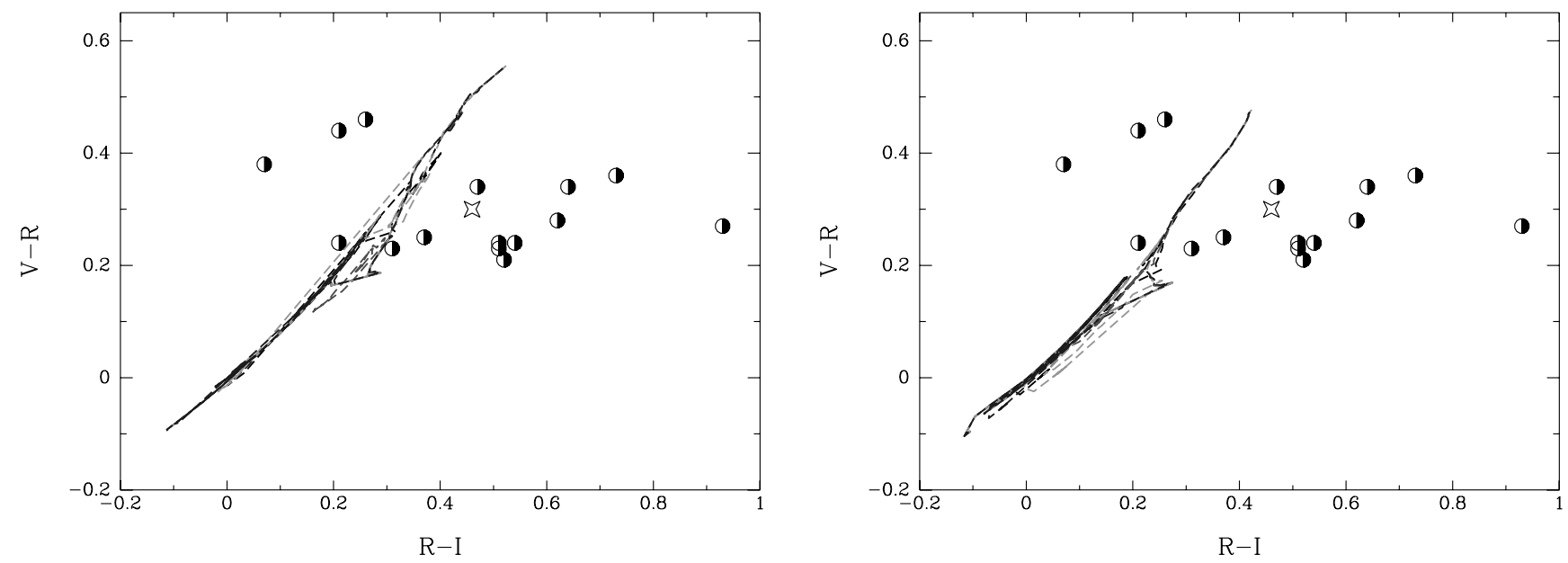

FIG. 6.-Both panels represent the evolution in the $(V-R)-(R-I)$ plane of the models used here to describe the four observed LCBGs. The left panel shows the first set of models, while the right panel shows the metal-poor models. The local data are taken from the H II galaxy sample from Telles \& Terlevich (1997). The four-pointed star represents the average position of this $\mathrm{H}$ in galaxy sample. Other symbols are the same as in Fig. 5. [See the electronic edition of the Journal for a color version of this figure.]

because of their low surface brightnesses, and only broad ranges are reported in the literature, mainly based in photometric measurements (see, e.g., Rakos et al. 2003; Jerjen et al. 2004). In the former work, the $[\mathrm{Fe} / \mathrm{H}]$ metallicity of $\mathrm{dE}$ galaxies is found to lie in the $1 / 100$ to 1.0 solar interval, while in the latter work the iron content of $\mathrm{dE}$ galaxies is found to range from $1 / 25$ to $1 / 3$ of the solar value. These broad ranges prevent us from using these values to discriminate between the presented models. If solar proportions are assumed, the previous iron content ranges roughly translate into $12+\log \mathrm{O} / \mathrm{H}$ ranging from 7.0 to 8.5 . This range is unfortunately in agreement with the metallicities of both model set 1 and model set 2 .

It is also seen in Figure 5 that, even though BCDGs are less luminous than $\mathrm{Sph}$ systems or $\mathrm{H}$ II galaxies, their $B-R$ colors are similar, although somewhat bluer on average. BCDGs are as luminous as the brightest globular clusters, and their stellar populations are also very old, like those of Sph systems. If they are the descendants of a hypothetical class of low-luminosity LCBGlike galaxies in the burst phase, the blue absolute magnitude of these objects should be around -16.0 . Such objects would be very hard to observe, and they would be very similar to dwarf irregular systems like NGC 1569 or NGC 1705.

Figure 5 shows that the underlying stellar populations of the local H II galaxies are compatible with the evolution of LCBGs in the sense that their $B-R$ colors were the same at some point in the past, around the time when the observed starbursts took place. It is then likely that the underlying stellar populations found in the presented LCBGs are much younger than the underlying stellar generations found in local $\mathrm{H}$ II galaxies. The underlying stellar population of local $\mathrm{H}$ II galaxies is found to be older that 1 Gyr by Westera et al. (2004). The older stellar generations derived in the simple models presented here resemble a somewhat aged intermediate-age population, again in the nomenclature given in Westera et al. (2004).

In order to compare in greater detail the stellar populations of LCBGs and $\mathrm{H}$ II galaxies, the evolution in the $(V-R)-(R-I)$ plane of the models devised here is presented in Figure 6 , together with the location of the underlying stellar populations of the 15 bright, high equivalent width $\mathrm{H}$ II galaxies from Telles \& Terlevich (1997). These latter objects are again represented by half-filled circles. The $V-R$ colors are in a very narrow range around 0.3 ; the $R-I$ color has a much greater range of variability, from 0 to 1 . This figure also shows that the $V-R$ color of the underlying population found in local $\mathrm{H}$ II galaxies is indeed attainable by LCBGs at some point during their evolution. ${ }^{23}$ The underlying stellar populations of local $\mathrm{H}$ in galaxies are then found to emit more power in the $I$ band than intermediate-redshift LCBGs of similar $V-R$ colors. This might be caused by the fact that intermediate-redshift LCBGs probably harbor lower relative numbers of red giant stars than the redder $\mathrm{H}$ in galaxies, since they have had much less time to evolve since the onset of the red giant phase of the evolution of their dominant stellar populations. Such very luminous red giant stars, existing in the extensions and wings of the local $\mathrm{H}$ II galaxies, would be responsible for a sizable fraction of the $I$-band luminosity of local $\mathrm{H}$ II galaxies.

These suggestions can be interpreted in light of the picture for $\mathrm{H}$ II galaxies presented in Westera et al. (2004), in which H II galaxies are a mixture of three populations. The first of them is the youngest population responsible for the observed emission lines; the second population is an intermediate-age generation, older than 50 Myr but younger than a few hundred megayears; and the third population is the oldest one, older than 1 Gyr. The single underlying stellar population used here can be thought to represent a mixture of the second and third populations of the Westera et al. (2004) model. As such, it can only accurately reproduce the colors of the real mixture it represents in a limited wavelength interval. Figure 6 shows that the model fails in the $I$ band. It is then natural to think that the real stellar mixture in $\mathrm{H}$ II galaxies is relatively richer in very old, $I$-emitting stars, namely, red giants. This very old and important stellar component has already been shown to exist in local $\mathrm{H}$ II galaxies. In addition to this, the work presented in Raimann et al. (2000) showed a detailed statistical analysis of the stellar populations found in a large sample of emission-line galaxies. It was found that a significant fraction of the stellar mass built in these systems is at least 500 Myr old. Present-day descendants of LCBGs will then have very old stellar populations, similar to the oldest stellar populations described in Westera et al. (2004). Should these systems undergo another star formation episode, they would be very similar

\footnotetext{
${ }^{23}$ In particular, according to model set 1 , the underlying populations of LCBGs presented such $V-R$ colors $4.9 \mathrm{Gyr}$ ago, when the observed starbursts took place. In the case of the metal-poor models, this happened $4.5 \mathrm{Gyr}$ ago, just after the observed starbursts occurred. The first set of models reached the average $R-I$ of the local sample $2.3 \mathrm{Gyr}$ ago, while model set 2 will attain such $R-I$ color in the future.
} 

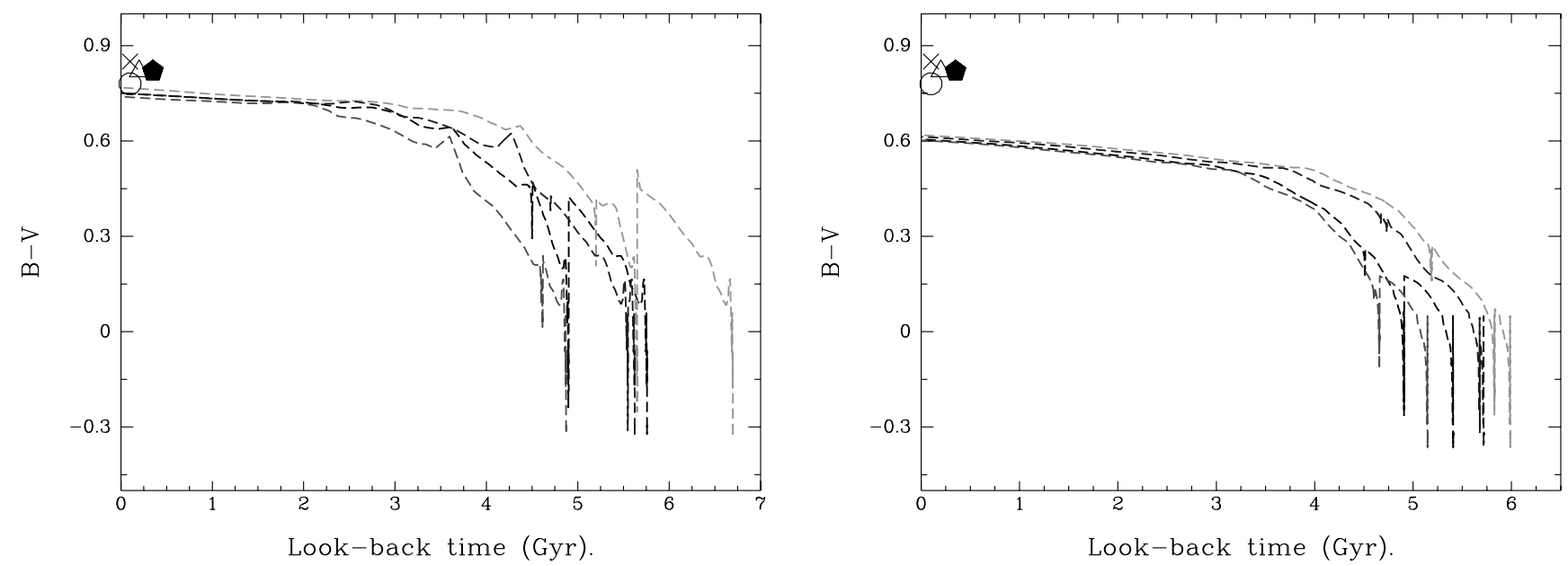

FIG. 7.-Temporal evolution of the stellar $B-V$ color with look-back time. The left panel shows the first set of models, while the right panel shows the metal-poor models. The symbols represent the $B-V$ color of several well-known local spheroidal systems. The pentagon represents NGC 205, the triangle represents IC 3393 , the cross represents NGC 3605, and the circle represents NGC 147. Other symbols are the same as in Fig. 5. [See the electronic edition of the Journal for a color version of this figure.]

to modern $\mathrm{H}$ II galaxies. More modeling and observational work is clearly needed in order to ascertain this.

Figure 7 presents other aspects of the evolution of the constructed models. It shows the evolution of the stellar $B-V$ color as a function of look-back time. The symbols at $t \sim 0$ represent four well-known spheroidal systems, as indicated in the figure. The metal-rich models end their evolution near the position occupied by the $\mathrm{dE} / \mathrm{Sph}$ sources. It can also be seen that the second set of models does not reach the very red colors of the presented Sph galaxies. Moreover, these metal-poor models can only reach $B-V=0.7$ in the distant future. Such colors are not in agreement with the colors of the handful of Sph galaxies presented for comparison.

In Figure 8 the evolution of the presented models in the $\log \left(M / L_{B}\right)-(B-V)$ diagram is shown. The positions of the LMC and individual dwarf elliptical systems are shown with specific symbols for each object. The positions of the LCBGs observed with the WFPC2 in Guzmán et al. (1998) are depicted using squares with asymmetric error bars. The errors in mass-to-light ratio arise from the fact that the masses used in Guzmán et al. (1998) were

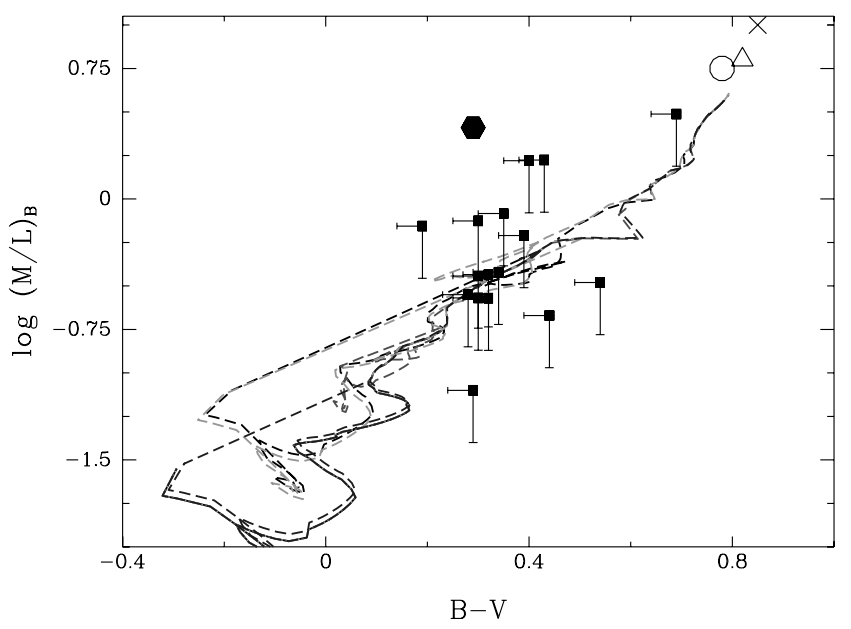

not stellar masses but dynamical ones. The presented error bars allow for a factor of 2 difference in both numbers. The errors in the $B-V$ color come from the contribution of the emission lines to the spectrum. This contribution has been assumed to be $0.05 \mathrm{mag}$ toward bluer colors for all objects. The $B-V$ color of the LMC has been corrected for the contribution of the line-emitting region, starburst 30 Dor, and extinction by subtracting 0.15 from the color.

It is again seen that model set 1 ends its evolution much closer to the Sph galaxies, although neither model falls neatly within the region defined by the Sph galaxies shown. The fact that the mass-to-light ratios of the first set of models are more similar to those of the Sph systems than those of model set 2 allows us to think that the other colors of dEs will be more similar to the colors predicted by model set 1 . It is also the case that the stellar populations of the presented models have a mass-to-light ratio lower than that of the LMC by the time their $B-V$ color is similar. This suggests that star formation has proceeded for a longer period of time in the LMC than in LCBGs, which is again consistent with LCBGs being observed at very high look-back times.

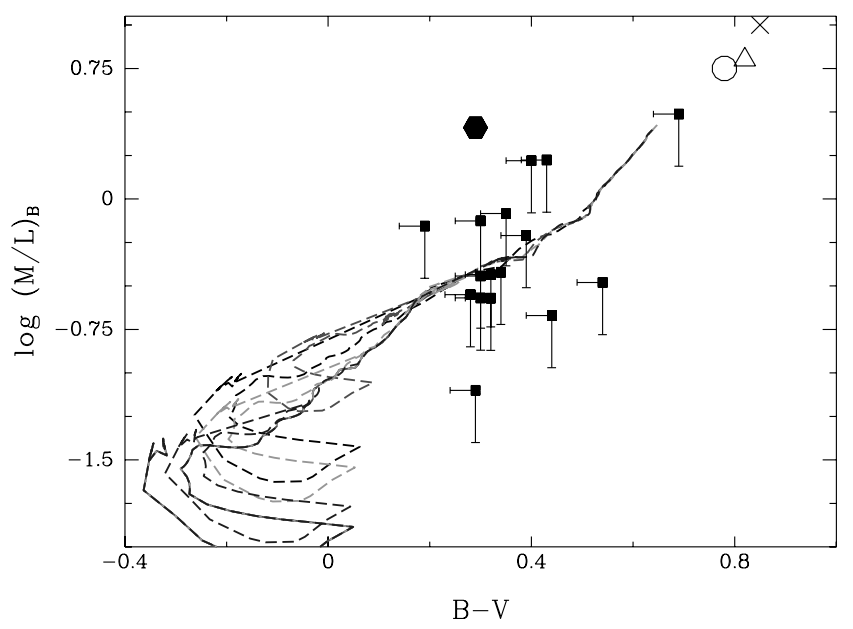

FIG. 8.- Model evolution in the $(B-V)-\left(M / L_{B}\right)$ plane. The left panel shows the first set of models, while the right panel shows the metal-poor models. The positions of the galaxies with mass-to-light ratios taken from Guzmán et al. (1998) are shown as squares, and their error bars are explained in the text. The other symbols represent the positions of several well-known local spheroidal systems. The LMC is included also. The triangle represents IC 3393, the cross represents NGC 3605, the circle represents NGC 147, and the hexagon represents the LMC. Other symbols are the same as in Fig. 5. [See the electronic edition of the Journal for a color version of this figure.] 

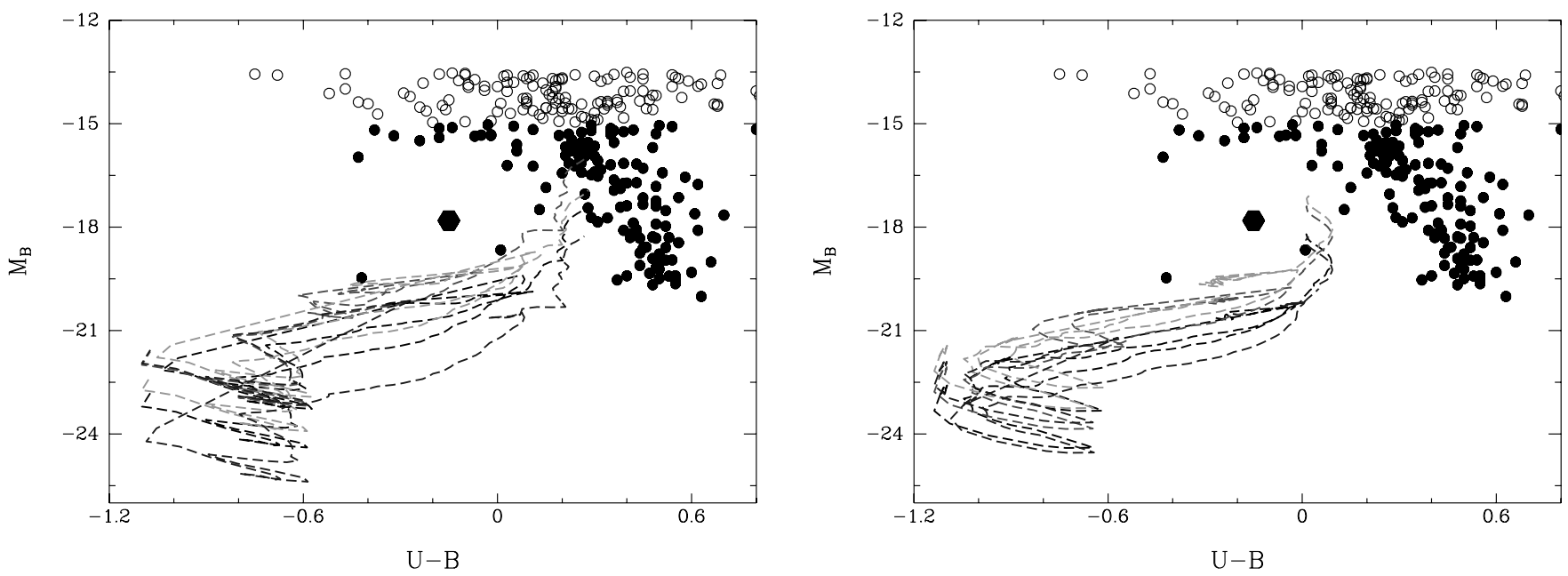

Fig. 9.- Model evolution in the $(U-B)-M_{B}$ plane. The LMC is included (hexagon). Both open and filled circles represent the Sph galaxies from Guzmán (1994). Filled circles represent objects brighter than $M_{B}=-15.0$. Open circles represent the dimmer objects. [See the electronic edition of the Journal for a color version of this figure.]

The Guzmán et al. (1998) objects cluster around the model tracks. In fact, six objects lie directly on top of the models themselves. This is reassuring in the sense that the bulk of the stellar inventory in the Guzmán et al. (1998) sources is probably well represented by the simple models presented here. However, it is somewhat unfortunate that the Guzmán et al. (1998) points fall both above and below the model lines, because this makes it impossible to further resolve the underlying stellar population. If, for instance, the Guzmán et al. (1998) points were all above the model tracks around the $\mathrm{LMC}$, it would have been possible to argue that the real underlying stellar population should be older than the age predicted by the models. This fact, however unfortunate, was to be expected, since the $B-V$ color is not very informative in the age range studied. The use of redder colors would be needed in order to gain this drop of information on the underlying stellar population.

It is also interesting to compare Figure 8 with Guzmán et al. (1998, Fig. 3). It is seen that the path of the models built in this work in the $\log \left(M / L_{B}\right)-(B-V)$ plane is very similar to the path of the Guzmán et al. (1998) models, implying that the differences in the age of the underlying stellar generation and relative mass of the ionizing population have little impact on the $B-V$ color and mass-to-light ratio, at least within these limits. Other observables will surely be more affected.

Figure 9 is very similar to Figure 5 . It shows how the presented models evolve in the $M_{B}-(U-B)$ plane. This time, only the positions of the LMC and the sample of spheroidal systems taken from Guzmán (1994) are included. The H ir galaxies and BCDGs are not included because there are no $U$-band data for these galaxies, and estimating them from $V$-band data would be affected by large uncertainties. The contribution to the $U-B$ color from the 30 Doradus cluster and extinction is assumed to be 0.1 . It is again seen that the final stage of the first set of models is much more similar to the observed properties of the Sph galaxies. Furthermore, all the models converge toward a point around $U-$ $B=0.3, M_{B}=-17.0$. This suggests that a Sph descendant of the distant LCBGs should be very red and luminous. This latter conclusion is weaker in Figure 5. However, since the model used is very simple, it is also likely that the aforementioned convergence toward this subset of the $\mathrm{Sph}$ galaxies merely reflects a weakness of the models. Indeed, should a major star-forming episode happen in these objects, the final position of the evolved
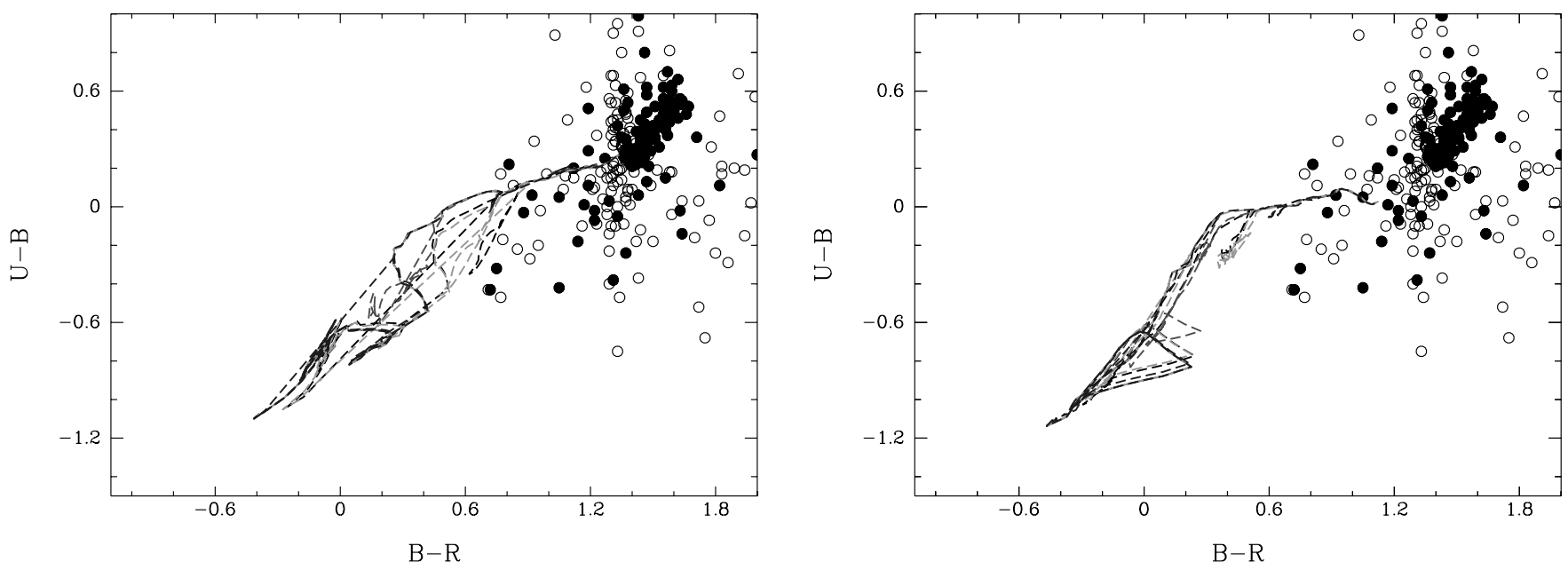

FIG. 10.-Evolution in the $(U-B)-(B-R)$ plane of the stellar populations that best represent the observed LCBGs. Both open and filled circles represent the Sph galaxies from Guzmán (1994). Filled circles represent objects brighter than $M_{B}=-15.0$. Open circles represent the dimmer objects. The panel layout is the same as in Fig. 5. [See the electronic edition of the Journal for a color version of this figure.] 
LCBGs would be different. It is also interesting to note that the LCBG models run very near the LMC at some point in the past, some time after the observed starbursts took place. A larger sample of LCBGs with more data would be needed to study the reality of the convergence effect mentioned above.

Finally, Figure 10 shows the evolution of the stellar populations predicted by our models in the $(U-B)-(B-R)$ plane. It is again seen that the first set of models ends its evolution much closer to the Sph galaxies than the metal-poor ones. Moreover, the models converge toward the bulk of the dwarf elliptical galaxies, and this conclusion also holds when only the bright Sph systems are considered, strengthening the idea that the Sph descendents of LCBGs are the more luminous ones.

\section{SUMMARY AND CONCLUSIONS}

We have used spatially resolved STIS long-slit spectroscopy together with optical and NIR imaging to investigate the stellar populations found in the line-emitting region of four LCBGs at intermediate $z$. This is one of the first times this has been done for distant, compact galaxies. The starbursting episodes are found to be less than 3.5 Myr old in most cases, with typical masses in the range from $5 \times 10^{6}$ to $12 \times 10^{6} M_{\odot}$. The mass contribution of the newly formed stars to the total mass of the stellar component ranges from $0.1 \%$ to $4 \%$, with an average value of $1 \%$. The ages of the older stellar generations found in these systems are in the 20-1400 Myr range, with an average value of 700 Myr. The NIR observations were used to check the optically derived stellar populations for consistency. It turns out that the agreement is generally very good, implying that the real stellar content is bound to be very similar to the two population mixtures used here. The lineemitting region spans $\sim 2 \mathrm{kpc}$ in size and is found to be very concentrated in the observed galaxies, although it is not always in the center of the optical galaxies. The metal content of the ionized gas phase cannot be readily determined with the data at hand, although the nitrogen measurements of very similar objects suggest slightly subsolar abundances. In addition, the NICMOS data allow us to regard the metal-rich models as the favored ones.

Although the available data are very limited, we have shown evidence for a very important underlying stellar population even in the central region of these sources, where the starburst completely dominates the observed optical emission. This population is somewhat evolved and accounts for most of the mass of these systems, despite the high look-back times probed. It can be said that the presented star formation histories are similar to some extent to the star formation histories of $\mathrm{H}$ II galaxies described in Westera et al. (2004). Both H II galaxies and LCBGs are, therefore, age-composite systems. They both have an old stellar population, together with a young stellar generation. It has also been found that, should LCBGs finally evolve into dwarf elliptical galaxies, their metal content must be at the higher end of the interval allowed by their observed line ratios. Unfortunately, given the broad range of metallicity determinations of Sph systems, this information cannot be used to help rule out any one of the presented models. The NIR data point toward the higher metallicity ones, however. In addition, it has been concluded that the Sph descendants of LCBGs can only be the more luminous and redder of such systems. The comparison of the LCBG models with the underlying population of luminous and local $\mathrm{H}$ II galaxies indicates that the star formation histories of the observed LCBGs and local $\mathrm{H}$ ir galaxies are similar. It has to be kept in mind, however, that $\mathrm{H}$ II galaxies probably have a larger relative number of red giant stars, as hinted by their enhanced power in the $I$ band. This can be simply interpreted by the fact that they are, arguably, 5 Gyr older than the more distant LCBGs. The observed differences in the redder colors probably arise from differences in the relative numbers of red giant stars. This suggests that the recent star formation histories of LCBGs and $\mathrm{H}$ II galaxies are similar up to some point. From that point backward, the star formation histories probably differ. We have also pointed out the possible existence of dwarf irregular systems in the intermediate-redshift range.

This work has been partially supported by Spanish DGCyT grant AYA-2004-08260-CO3 and Spanish MECD FPU grant AP2000-1389. Partial support from the Comunidad de Madrid under grant S0505/ESP/000237 (ASTROCAM) is acknowledged. A. I. D. acknowledges support from the Spanish MEC through sabbatical grant PR2006-0049 and is grateful for the hospitality of the Institute of Astronomy of Cambridge. R. G. acknowledges funding from NASA/STScI grant HST GO 08678.04-A and LTSA NAG5-11635. M. A. B. also acknowledges support from NASA/STScI grants HST GO 8678, GO 9126, GO 7675, and AR-9917 and NSF AST 06-07516. We also thank an anonymous referee for help in improving the readability and scientific quality of the text.

\section{REFERENCES}

Allende-Prieto, C., Lambert, D. L., \& Asplund, M. 2001, ApJ, 556, L63

Balzano, V. A. 1983, ApJ, 268, 602

Bershady, M. A., Vils, M., Hoyos, C., Guzmán, R., \& Koo, D. C. 2005, in Starbursts: From 30 Doradus to Lyman Break Galaxies, ed. R. de Grijs \& R. M. González Delgado (Dordrecht: Springer), 177

Bruzual, G., \& Charlot, S. 2003, MNRAS, 344, 1000

Burstein, D., \& Heiles, C. 1982, AJ, 87, 1165

Caon, N., Cairós, L. M., Aguerri, J. A. L., \& Muñoz-Tuñón, C. 2005, ApJS, 157,218

Cerviño, M., Gómez-Flechoso, M. A., Castander, F. J., Schaerer, D., Mollá, M., Knödlseder, J., \& Luridiana, V. 2001, A\&A, 376, 422

Cerviño, M., Luridiana, V., \& Castander, F. J. 2000, A\&A, 360, L5

Cerviño, M., Valls-Gabaud, D., Luridiana, V., \& Mas-Hesse, J. M. 2002, A\&A, 381,51

de Grijs, R., Gilmore, G. F., Mackey, A. D., Wilkinson, M. I., Beaulieu, S. F., Johnson, R. A., \& Santiago, B. X. 2002, MNRAS, 337, 597

Denicoló, G., Terlevich, R., \& Terlevich, E. 2002, MNRAS, 330, 69

Díaz, A. I., Álvarez, M. Á., Terlevich, E., Terlevich, R., Portal, M. S., \& Aretxaga, I. 2000a, MNRAS, 311, 120

Díaz, A. I., Castellanos, M., Terlevich, E., \& Luisa García-Vargas, M. 2000b, MNRAS, 318, 462

Dolphin, A. E. 2000, PASP, 112, 1397
Doublier, V., Caulet, A., \& Comte, G. 1999, A\&AS, 138, 213

Doublier, V., Comte, G., Petrosian, A., Surace, C., \& Turatto, M. 1997, A\&AS, 124,405

Edmunds, M. G., \& Pagel, B. E. J. 1984, MNRAS, 211, 507

Fukugita, M., Shimasaku, K., \& Ichikawa, T. 1995, PASP, 107, 945

Guzmán, R. 1994, Ph.D. thesis, Univ. Durham

Guzmán, R., Gallego, J., Koo, D. C., Phillips, A. C., Lowenthal, J. D., Faber, S. M., Illingworth, G. D., \& Vogt, N. P. 1997, ApJ, 489, 559

Guzmán, R., Jengren, A., Koo, D. C., Bershady, M. A., \& Simard, L. 1998, ApJ, 495, L13

Guzmán, R., Koo, D. C., Faber, S. M., Illingworth, G. D., Takamiya, M., Kron, R. G., \& Bershady, M. A. 1996, ApJ, 460, L5

Hammer, F., Gruel, N., Thuan, T. X., Flores, H., \& Infante, L. 2001, ApJ, 550, 570

Hoyos, C., \& Díaz, A. I. 2006, MNRAS, 365, 454

Hoyos, C., Guzmán, R., Bershady, M. A., Koo, D. C., \& Díaz, A. I. 2004, AJ, 128,1541

Jerjen, H., Binggeli, B., \& Barazza, F. D. 2004, AJ, 127, 771

Kennicutt, R. C., Jr., Tamblyn, P., \& Congdon, C. E. 1994, ApJ, 435, 22

Kewley, L. J., \& Dopita, M. A. 2002, ApJS, 142, 35

Kobulnicky, H. A., \& Zaritsky, D. 1999, ApJ, 511, 118

Koo, D. C., Bershady, M. A., Wirth, G. D., Stanford, S. A., \& Majewski, S. R. 1994, ApJ, 427, L9 
Koo, D. C., Guzmán, R., Faber, S. M., Illingworth, G. D., Bershady, M. A., Kron, R. G., \& Takamiya, M. 1995, ApJ, 440, L49

Leitherer, C., et al. 1999, ApJS, 123, 3

Lilly, S. J., Tresse, L., Hammer, F., Crampton, D., \& Le Fevre, O. 1995, ApJ, 455,108

Loose, H.-H., \& Thuan, T. X. 1986, ApJ, 309, 59

Maeder, A. 1992, A\&A, 264, 105

Maeder, A., \& Meynet, G. 2001, A\&A, 373, 555

Mamon, G. A., \& Lokas, E. L. 2005, MNRAS, 363, 705

Markarian, B. E. 1967, Astrofizika, 3, 24

McGaugh, S. 1991, ApJ, 380, 140

Navarro, J. F., et al. 2004, MNRAS, 349, 1039

Osterbrock, D. E. 1989, Astrophysics of Gaseous Nebulae and Active Galactic Nuclei (Mill Valley: University Science Books)

Pagel, B. E. J., Edmunds, M. G., Blackwell, D. E., Chun, M. S., \& Smith, G. 1979, MNRAS, 189, 95

Pagel, B. E. J., Edmunds, M. G., \& Smith, G. 1980, MNRAS, 193, 219

Papaderos, P., Loose, H.-H., Fricke, K. J., \& Thuan, T. X. 1996a, A\&A, 314, 59

Papaderos, P., Loose, H.-H., Thuan, T. X., \& Fricke, K. J. 1996b, A\&AS, 120, 207
Phillips, A. C., Guzmán, R., Gallego, J., Koo, D. C., Lowenthal, J. D., Vogt, N. P., Faber, S. M., \& Illingworth, G. D. 1997, ApJ, 489, 543

Pilyugin, L. S. 2000, A\&A, 362, 325

Raimann, D., Bica, E., Storchi-Bergmann, T., Melnick, J., \& Schmitt, H. 2000, MNRAS, 314, 295

Rakos, K., Schombert, J., Odell, A., \& Maitzen, M. 2003, Ap\&SS, 284, 803

Rosa-González, D., Terlevich, E., \& Terlevich, R. 2002, MNRAS, 332, 283

Sandage, A., \& Tammann, G. 1974, ApJ, 190, 525

Sargent, W. L. W., \& Searle, L. 1970, ApJ, 162, L155

Searle, L., \& Sargent, W. L. W. 1972, ApJ, 173, 25

Stolte, A., Brandner, W., Grebel, E. K., Lenzen, R., \& Lagrange, A.-M. 2005, ApJ, 628, L113

Telles, E., Melnick, J., \& Terlevich, R. 1997, MNRAS, 288, 78

Telles, E., \& Terlevich, R. 1997, MNRAS, 286, 183

Terlevich, A. I., Caldwell, N., \& Bower, R. G. 2001, MNRAS, 326, 1547

Terlevich, R., Melnick, J., Masegosa, J., Moles, M., \& Copetti, M. V. F. 1991, A\&AS, 91, 285

Westera, P., Cuisinier, F., Telles, E., \& Kehrig, C. 2004, A\&A, 423, 133

Whitford, A. E. 1958, AJ, 63, 201

Zwicky, F. 1965, ApJ, 142, 1293 